\title{
Dissecting the pathobiology of altered MRI signal in amyotrophic lateral sclerosis: A post mortem whole brain sampling strategy for the integration of ultra-high-field MRI and quantitative neuropathology
}

Menuka Pallebage-Gamarallage ${ }^{1 *} \mathbb{0}$, Sean Foxley ${ }^{1,2,3}$, Ricarda A. L. Menke ${ }^{1,2}$, Istvan N. Huszar ${ }^{1,2}$, Mark Jenkinson ${ }^{1,2}$, Benjamin C. Tendler ${ }^{1,2}$, Chaoyue Wang ${ }^{1,2}$, Saad Jbabdi ${ }^{1,2}$, Martin R. Turner ${ }^{1,2}$, Karla L. Miller ${ }^{1,2}$ and Olaf Ansorge 1* $^{*}$

\begin{abstract}
Background: Amyotrophic lateral sclerosis (ALS) is a clinically and histopathologically heterogeneous neurodegenerative disorder, in which therapy is hindered by the rapid progression of disease and lack of biomarkers. Magnetic resonance imaging (MRI) has demonstrated its potential for detecting the pathological signature and tracking disease progression in ALS. However, the microstructural and molecular pathological substrate is poorly understood and generally defined histologically. One route to understanding and validating the pathophysiological correlates of MRI signal changes in ALS is to directly compare MRI to histology in post mortem human brains.

Results: The article delineates a universal whole brain sampling strategy of pathologically relevant grey matter (cortical and subcortical) and white matter tracts of interest suitable for histological evaluation and direct correlation with MRI. A standardised systematic sampling strategy that was compatible with co-registration of images across modalities was established for regions representing phosphorylated 43-kDa TAR DNA-binding protein (pTDP-43) patterns that were topographically recognisable with defined neuroanatomical landmarks. Moreover, tractographyguided sampling facilitated accurate delineation of white matter tracts of interest. A digital photography pipeline at various stages of sampling and histological processing was established to account for structural deformations that might impact alignment and registration of histological images to MRI volumes. Combined with quantitative digital histology image analysis, the proposed sampling strategy is suitable for routine implementation in a high-throughput manner for acquisition of large-scale histology datasets. Proof of concept was determined in the spinal cord of an ALS patient where multiple MRI modalities (T1,T2, FA and MD) demonstrated sensitivity to axonal degeneration and associated heightened inflammatory changes in the lateral corticospinal tract. Furthermore, qualitative comparison of R2* and susceptibility maps in the motor cortex of 2 ALS patients demonstrated varying degrees of hyperintense signal changes compared to a control. Upon histological evaluation of the same region, intensity of signal changes in both modalities appeared to correspond primarily to the degree of microglial activation.
\end{abstract}

\footnotetext{
*Correspondence: menuka.pallebagegamarallage@ndcn.ox.ac.uk; olaf. ansorge@ndcn.ox.ac.uk

1 Nuffield Department of Clinical Neurosciences, University of Oxford,

Oxford, UK

Full list of author information is available at the end of the article
} 
Conclusion: The proposed post mortem whole brain sampling methodology enables the accurate intraindividual study of pathological propagation and comparison with quantitative MRI data, to more fully understand the relationship of imaging signal changes with underlying pathophysiology in ALS.

Keywords: Amyotrophic lateral sclerosis, Magnetic resonance imaging, Post mortem brain, Systematic sampling, Histology, MRI-histology correlation

\section{Background}

Amyotrophic lateral sclerosis (ALS) is a typically rapidly progressive, fatal neurodegenerative disorder that is genetically and phenotypically heterogeneous. It is primarily characterised by selective degeneration of upper and lower motor neurons [1]. A significant proportion of ALS patients develop cognitive impairment within the spectrum of frontotemporal dementia (FTD) [2]. The diagnosis is essentially clinical [3]. Conventional magnetic resonance imaging (MRI) is generally used during diagnosis as a tool to exclude ALS mimics, but advanced techniques such as functional MRI and diffusion tensor imaging (DTI) have enabled investigation of both structural and functional connectivity in vivo [4]. Diffusion tensor imaging in particular has highlighted the widespread cerebral pathology associated with ALS [5]. Furthermore, quantitative susceptibility mapping has demonstrated potential as a biomarker of upper motor neuron dysfunction in ALS [6-8]. However, these MRI markers are non-specific and generally known to be influenced by several aspects of tissue related to neuropathology. It is therefore crucial to define the microstructural and molecular pathologic correlates of these MRI measures in ALS. Therefore, MRI-histology correlation analysis in post mortem tissue offers a platform to discover the histological underpinnings of MRI signal changes.

Limited studies have explored the relationship between post mortem MRI and histology in ALS with assessment restricted to a segment of the primary motor cortex of a hemisphere in small number of cases. Reported changes include R2* hyperintensity in the middle and deep layers of the grey matter and signal alteration in the subcortical white matter of the motor cortex [9]. Histopathological evaluation of the corresponding cortical region showed microglial iron accumulation and myelin pallor in subcortical white matter [9]. Similarly, diminished contrast between grey and white matter has been observed, with altered $\mathrm{T} 1$ relaxation rate ratio in the motor cortex corresponding to reduced neuronal and axonal density and increased astroglial density and reactivity [10]. The study also identified comparable differences in T1 relaxation rate ratio in other cortical regions anterior and posterior to the motor cortex as a gradient suggestive of contiguous pathology spread [11-13], though with limited histological evaluation. Interestingly, no significant changes in average $\mathrm{T} 2$ relaxation rate ratio were detected [10]. These MRI modalities are sensitive to more than one aspect of tissue related to neuropathology. For accurate interpretation of specificity and validation of the sensitivity of such findings, multiple MRI modalities accompanied with a range of histological parameters must be considered, extending to other regions involved in disease progression.

Combining whole brain post mortem MRI with systematic histological evaluation representing ALS-FTD pathology propagation is a pathway to determining and validating disease specific changes in structural organisation and connectivity. Whole fixed brain MRI confer additional benefits over MRI of small segmented regions, including reduced artefacts related to MRI, preserved landmarks for topographical identification of regions of interest, and a single scan that can cover the whole brain [14]. Given the growing interest, studies have established direct correlation between post mortem whole brain MRI in situ with large-scale digital histology on corresponding coronal slices for evaluation of pathology and structural connectivity [15-18]. However, whole slice digital histology is costly and labour-intensive, therefore routine implementation is not feasible on a large number of cases. Interestingly, precise comparison of high-resolution whole brain post mortem MRI with histological analysis on strategically sampled pathologically relevant regions of interest in multiple sclerosis have also been demonstrated $[14,19]$.

This article outlines a universal whole brain sampling strategy suitable for systematic evaluation of differential microstructural and molecular changes associated with pathology spread in cortical, subcortical and deep white matter regions of ALS and FTD to ultimately enable accurate post mortem MRI-histology correlation. The proposed methodology, modified from a standard brain banking protocol, was carefully designed to selectively sample pathologically relevant regions guided by anatomical landmarks and tractography. The protocol takes into consideration various aspects of downstream coregistration requirements and quantitative histological analyses to facilitate accurate depiction of changes within the regions of interest. 


\section{Methods}

\section{Methodological considerations}

Identification of neuroanatomical regions relevant to pathology spread

Identification of neuroanatomical regions that depict ALS and FTD pathology spread is crucial to establishing a universal whole brain sampling strategy by identifying clinical phenotypes and accompanying neuropathological characterisation. Clinical phenotypes of ALS are heterogeneous and the focality of symptom onset and subsequent contiguous spread of motor dysfunction are common to all diagnosed patients and indicative of a disease continuum [20]. Neuropathologically, ALS is characterised by a degree of upper and lower motor neuron loss concomitant with degeneration of their respective axonal projections, glial activation and the pathological aggregation of 43-kDa TAR DNA-binding protein (TDP-43) [21, 22]. The unique molecular feature of TDP-43 proteinopathy is reported in almost all cases of ALS and in the largest subset of FTD supporting the concept of ALS-FTD as a pathological spectrum $[12,23]$. Although neuropathological evaluation is made at the "end-stage" of disease, recent studies have defined a pathological staging system based on systematic neuroanatomical distribution of TDP-43 pathology that correlated with severity of clinical phenotypes [11, 12, 24, 25].

In a system of ALS pathological 'stages' based on regional patterns of post mortem phosphorylated TDP43 (pTDP-43) pathology, Stage 1 was defined as involving the upper motor neurons of the primary motor cortex and the lower motor neurons of the brainstem and the spinal cord [25]. From the motor cortex, Stage 2 involves pathology extended rostrally to prefrontal cortical regions of the middle frontal gyrus and caudally to the reticular formation of the brainstem, red nucleus and precerebellar nuclei. Stage 3 was defined as pTDP-43 lesions extending into the gyrus rectus and orbital gyri of the basal prefrontal cortex, post-central sensory areas of the parietal lobe, the temporal lobe and the striatum. Stage 4 involves pathological burden in the anteromedial regions of the temporal lobe and the hippocampus. Distinct pTDP-43 distribution patterns have also been proposed for behavioural variant FTD (bvFTD) [12], the most common form of FTD overlapping with ALS [26]. In bvFTD, pTDP-43 lesions first appear in the basal prefrontal cortex (orbital gyri and gyrus rectus) and amygdala in Pattern I. Involvement caudally into the prefrontal cortical regions (middle frontal gyrus, insular cortex and anterior cingulate), temporal lobe, including hippocampus, and subcortical regions striatum, thalamus, red nucleus and precerebellar nuclei of pons and medulla defines Pattern II. In Pattern III, motor and parietal cortical regions and lower motor neurons of the brainstem and spinal cord are involved. In contrast to ALS, Pattern IV in bvFTD involves pTDP-43 within the occipital pole [12].

The concept of anterograde corticofugal propagation of pTDP-43 is supported by these patterns and the presence of pTDP-43 pathology in the subcortical white matter of the affected cortical areas and the involvement of subcortical regions with substantial neocortical afferents $[11,25,27]$. Diffusion tensor imaging has been utilised to analyse white matter tracts that are likely to be involved in corresponding pTDP-43-based stages of ALS in vivo [28]. The study observed substantial differences in corticospinal tract (Stage 1), corticorubral and corticopontine tracts (Stage 2), corticostriatal pathway (Stage 3) and proximal portion of perforant pathway (Stage 4) in ALS in comparison to the control group and tract involvement corresponded with disease duration. Experimental evidence in vivo also supports involvement of axonal pathways in FTD. Diffusion tensor imaging of bvFTD showed significant degree of white matter damage to the uncinate fasciculus, inferior and superior longitudinal fasciculus, genu of corpus callosum, forceps minor and cingulum bundle [29-31]. Furthermore, the fornix was identified as a key locus of damage in bvFTD [32]. However, a more limited number of studies have validated histopathological changes in deep white matter tracts with demonstrated involvement in ALS pathology in vivo. Independent neuropathological validation demonstrated a lack of pTDP-43 pathology in corticospinal tract, corpus callosum and cingulum bundle in ALS staging [27]. Interestingly, other distinctive pathological features such as glial activation and axonal degeneration in corticospinal tract and corpus callosum have been reported previously [33-36], and callosal involvement is one of the most consistent MRI findings in ALS [37, 38]. These findings suggest that the underlying pathobiological features of neuroimaging modalities remain unclear highlighting the need for validation of MRI modalities with their respective histologic correlates at cellular and subcellular levels.

Validation of microstructural changes in regions representing pTDP-43 spreading pattern in post mortem brains against whole brain MRI signals requires systematic sampling of pathologically relevant regions of interest. Presented in Table 1 are the key neuroanatomical regions (cortical and subcortical grey matter; subcortical and deep white matter tracts) that (1) represent ALS and bvFTD pathology spread, and (2) are topographically recognisable with defined anatomical landmarks to facilitate systematic sampling and MRI-histology co-registration. 
Table 1 Regions of the brain involved in PTDP-43 staging system in ALS and bvFTD

\begin{tabular}{lll}
\hline Regions of interest & ALS & bvFTD \\
\hline $\begin{array}{l}\text { Grey matter } \\
\text { Precentral gyrus (motor cortex) }\end{array}$ & Stage 1 & Pattern III \\
$\begin{array}{l}\text { Brainstem } \\
\text { Hypoglossal nucleus }\end{array}$ & Stage 1 & Pattern III \\
Precerebellar nuclei/red nucleus & Stage 2 & Pattern II \\
Middle frontal gyrus & Stage 2 & Pattern II \\
Cingulate-anterior & Stage 2/3 & Pattern II/II \\
Postcentral gyrus (somatosensory cortex) & Stage 3 & Pattern III \\
Orbitofrontal cortex & & \\
Orbital gyrus & Stage 3 & Pattern I \\
Gyrus rectus & Stage 3 & Pattern I \\
Striatum & Stage 3 & Pattern II \\
Hippocampal formation & Stage 4 & Pattern II \\
Occipital pole & Not affected & Pattern IV \\
White matter tracts & & \\
Corticospinal tract & Stage 1* & Pattern III* \\
Cingulum & Not affected [27] & Affected* \\
Fornix & Not assessed & Affected* \\
Corpus callosum & Affected* & Affected* \\
\hline
\end{tabular}

The table highlights brain regions that have been described in the post mortem four stage hypothesis of pTDP-43 [12, 25], or demonstrated to be involved in the disease process in vivo

* White matter tract changes demonstrated with in vivo MR imaging, however, independent of pTDP-43 validation or in the absence of pathology [27, 39]

\section{Systematic sampling and MRI-histology co-registration requirements}

Standardised systematic sampling should be implemented for making valid statements about regions of interest, however, challenged by the highly folded nature of the cortex, large number of areas with variable sizes and undeniable inter-individual variability [40]. Nevertheless, maintaining reproducibility with neuroanatomical accuracy is crucial to a standardised sampling strategy in a disease specific cohort. The proposed sampling strategy considers two approaches to minimise interindividual variability whilst maintaining neuroanatomical accuracy: (1) the use of neuroanatomical landmarks, e.g. gyri, sulci and subcortical grey matter that can be robustly identified in all brains on MRI and during cut-up of the corresponding brain, for identifying cortical and subcortical regions of interest and (2) the use of diffusion MRI tractography to guide identification and sampling of white matter tracts. These approaches enabled implementation of systematic sampling for regions of interest to provide accurate estimates of changes in them [41] and are aimed to facilitate multi-modal alignment and coregistration of 2-dimensional (2-D) histological images on 3-dimensional (3-D) MR volumes [42, 43].
Direct MRI-histology correlation, in vivo and ex vivo, is generally made difficult by the heterogeneity in characteristics on images from the two modalities in addition to the deformation and damage caused by histological processing [42]. Although substantial distortions are likely to occur at the time of fresh brain extraction and during fixation, geometric deformations also occur during MRI scanning due to the brain being stabilised in a closefitting container resulting in twisting or compression. Subsequent serial sampling of the fixed brain following scanning, histological sectioning of the brain regions into 2-D slices and staining would often introduce tears, rips, folds, missing pieces of tissue, artefacts, debris, uneven staining gradient and displacement of anatomical landmarks such as gyri and sulci from the original 3-D geometry $[40,43,44]$. In addition, tissue shrinkage as a result of chemical processing for paraffin embedding needs to be taken into account. Several studies have explored registration of histology to MR with digital photographs of block faces acquired during sampling, in addition to stained sections, to serve as an intermediate modality for alignment and reconstruction on a 3-D volume [40, 4347]. To enable accurate co-registration whilst taking into consideration structural deformations and damage, the proposed sampling strategy is coupled with digital photography pipeline (Fig. 1) at various stages of block face sampling and histological processing: (1) coronal slices (2) sampled regions of interest and their remnants, and (3) trimmed surfaces of paraffin blocks with (4) stained serial histological sections.

\section{Tissue preparation and MR imaging}

Post mortem tissues were obtained from the Oxford Brain Bank. Fixed whole brains were drained of formalin and immersed in $3 \mathrm{M}^{\mathrm{TM}}$ Fluorinert $^{\mathrm{TM}}$ (FC-3283) for susceptibility matching (Fluorinert has a similar susceptibility to tissue, however, no signal) in a closed fitting plastic mould and imaged with a Siemens $7 \mathrm{~T}$ scanner using 32-channel receive, single-channel transmit RF coil for $48 \mathrm{~h}$. Magnetic resonance imaging acquisition consisted of a set of protocols that provides a variety of contrasts (Table 2), including structural scans, relaxography (T1, T2), susceptibility-weighted contrast and diffusion weighted steady-state free precession (DW-SSFP) for more specific measures of white matter [48-50]. Following MRI, brain drained of Fluorinert ${ }^{\mathrm{TM}}$ and returned to formalin in preparation for sampling.

\section{MR image processing pipelines}

Structural datasets obtained with the 3-D - TRUFI protocol were averaged by calculating the root-mean-square of the individual phase-cycled scans. The T2-mapping protocol utilised a voxel-wise, signal-weighted 


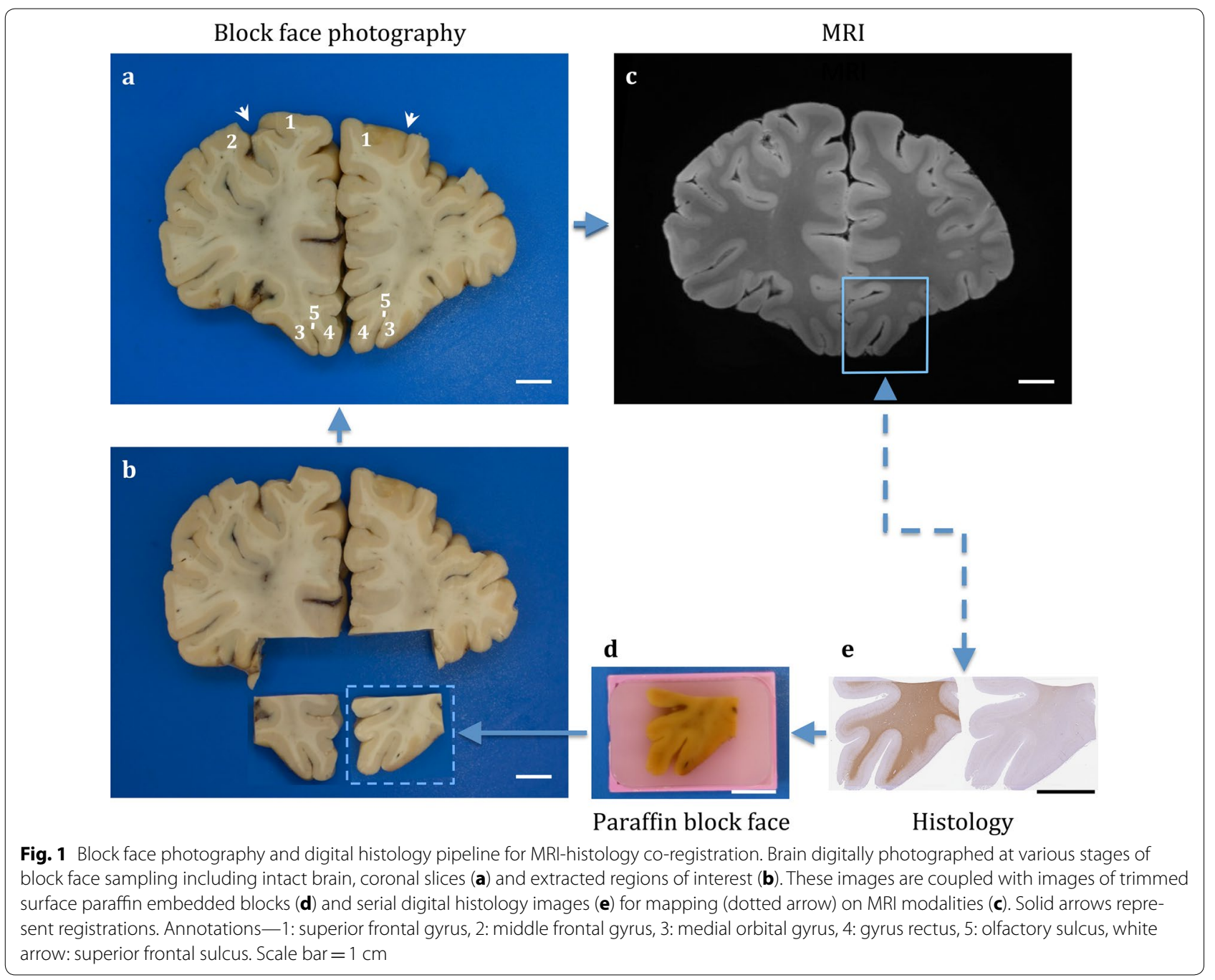

pseudoinverse of the linearised temporal signal evolution from the T2 turbo spin-echo protocol. An identical process was adopted to generate $\mathrm{T} 2 \%$-maps, fitting to the magnitude component of signal evolution from the susceptibility weighted imaging (SWI) protocol. The T1-mapping protocol utilised a non-linear least-squares fit to the signal evolution from the T1 turbo spin-echo protocol. All fitting was performed using the NumPy and SciPy toolboxes in Python [51, 52].

Processing of the diffusion data obtained via the DWSSFP imaging protocol utilised modified forms of DTIFIT and BEDPOSTX from the FSL toolbox [53-55], to account for the DW-SSFP $[48,56]$, dual flip-angle datasets. All co-registration between and within imaging modalities was performed using FSL-FLIRT $[57,58]$.

Phase datasets from the SWI protocol were processed to generate quantitative susceptibility maps in MATLAB (The MathWorks, Inc.), original phase images were first unwrapped using a Laplacian-based method [59], the unwrapped phase images were subsequently filtered using V-SHARP algorithm [60] to remove the background field, quantitative susceptibility maps were finally generated using STAR-QSM algorithm from the STI Suite toolbox [61].

\section{Sampling strategy for regions of interest}

Brains were prepared separately for sampling initially by carefully removing the meninges, photographed at several planes and dimensions were measured prior to dissection. Brain stem and cerebellum were removed from the cerebrum by knife section across the cerebral peduncle at the level of the 3rd cranial nerve (oculomotor nerve) in a plane perpendicular to the brainstem and aqueduct. Specific regions of interest were then sampled in both hemispheres as described in the following sections. 
Table 2 Whole brain MRI protocol parameters

\begin{tabular}{|c|c|}
\hline \multicolumn{2}{|l|}{ a. DW-SSFP } \\
\hline Lines per TR & 3 \\
\hline q value $\left(\mathrm{cm}^{-1}\right)$ & 300 \\
\hline$b_{\text {eff }}\left(s / m^{2}\right)$ & 5150 \\
\hline TE/TR (ms) & $21 / 28$ \\
\hline Resolution (mm) & $0.85 \times 0.85 \times 0.85$ \\
\hline Flip angle $\left(^{\circ}\right)$ & 24 and 94 \\
\hline Number of directions & 120 per flip angle \\
\hline Bandwidth (Hz/pixel) & 393 \\
\hline Duration 1 volume (min:s) & $5: 50$ \\
\hline Total duration (h:min:s) & $24: 30: 00$ \\
\hline \multicolumn{2}{|l|}{ b. Structural 3-D-Trufi } \\
\hline TE/TR (ms) & $4.09 / 8.16$ \\
\hline Resolution (mm) & $0.25 \times 0.25 \times 0.27$ \\
\hline Flip angle $\left(^{\circ}\right)$ & 30 \\
\hline Phase cycling $\left(^{\circ}\right)$ & $0,90,180,270$ \\
\hline Averages (per PC) & 2 \\
\hline Bandwidth (Hz/pixel) & 393 \\
\hline \multicolumn{2}{|l|}{ c. T1-map turbo spin-echo } \\
\hline TE/TR (ms) & $14.0 / 1000$ \\
\hline Resolution (mm) & $1.0 \times 1.0 \times 1.0$ \\
\hline Flip angle $\left(^{\circ}\right)$ & 90 and 180 \\
\hline Averages & 1 \\
\hline Bandwidth (Hz/pixel) & 130 \\
\hline Inversion times (ms) & $60,120,240,480,935$ \\
\hline \multicolumn{2}{|l|}{ d. T2-map turbo spin-echo } \\
\hline TEs (ms) & $25,38,50,63,76$ \\
\hline TR (ms) & 1000 \\
\hline Resolution (mm) & $1.0 \times 1.0 \times 1.0$ \\
\hline Flip angle $\left(^{\circ}\right)$ & 90 and 180 \\
\hline Averages & 1 \\
\hline Bandwidth (Hz/pixel) & 130 \\
\hline \multicolumn{2}{|c|}{ e. Susceptibility weighted imaging multi-gradient echo } \\
\hline TEs (ms) & $2,8.6,15.2,21.8,28.4,35$ \\
\hline TR (ms) & 38 \\
\hline Resolution (mm) & $0.5 \times 0.5 \times 0.6$ \\
\hline Flip angle $\left({ }^{\circ}\right)$ & 15 \\
\hline Number of repetitions & 4 \\
\hline Bandwidth (Hz/pixel) & 650 \\
\hline
\end{tabular}

\section{Motor and somatosensory cortex}

The motor cortex (ALS Stage 1) on the precentral gyrus is demarcated by the anatomical borders the precentral sulcus (anteriorly) and the central sulcus (posteriorly) on the dorsolateral surface. Somatosensory cortex was identified in the post-central gyrus posterior to the central sulcus. Sampling of the motor and somatosensory cortex was restricted to clinicopathologically relevant regions defined by the anatomical landmarks (Fig. 2):
1. Leg area was represented on the medial surface of the paracentral lobule at the banks of the interhemispheric fissure.

2. Motor hand area was distinguished by the hand knob (shaped as an inverted omega).

3. Face area was identified on the precentral gyrus lateral to the intersection of inferior frontal sulcus and precentral sulcus.

Approximately $1 \mathrm{~cm}$ blocks of tissue perpendicular to the central sulcus were dissected out at the leg area, middle hand knob region and face area to include pre-central gyrus, corresponding post-central gyrus, central sulcus and subcortical white matter (Fig. 2). Care was taken to ensure that blocks were sampled at a reasonable depth to maintain integrity of pre- and post-central gyri together with the central sulcus on the same block (to facilitate the identification of the M1/S1 border, which is in the depth of the sulcus).

\section{Coronal slices}

Following extraction of the primary motor and somatosensory cortical regions, brains were sliced in a coronal plane through the mammillary bodies perpendicular to the longitudinal axis of the forebrain. Subsequent coronal slices, at $1 \mathrm{~cm}$ intervals, were made anterior and posterior to the initial cut (Fig. 3). Anterior and posterior surfaces of each slice were photographed prior to sampling of the following regions of interest.

\section{Middle frontal gyrus}

The middle frontal gyrus is involved in Stage 2 and Pattern II of ALS and bvFTD, respectively. It forms part of the dorsolateral prefrontal cortex involved in regulation of attention, executive function and working memory that have been reported to be deficient in ALS patients without dementia [62-64]. The middle frontal gyrus is one of the largest regions in the prefrontal cortex located between the superior frontal sulcus, inferior frontal sulcus and pre-central sulcus [65]. It cytoarchitectonically constitutes Brodmann areas (BA) 6, 8, 9, 46 and 10 along its caudal-rostral axis [65, 66].

The middle frontal gyrus was identified in coronal slices lateral to the anatomical landmark superior frontal sulcus and sampled at a reasonable depth $(\sim 2 \mathrm{~cm})$ to include subcortical white matter, and the adjacent superior frontal gyrus and sulcus (Fig. 3). Sampling was carried out in serial coronal slices rostral to the precentral gyrus extending to the anterior most slice that clearly defines the superior frontal sulcus. 

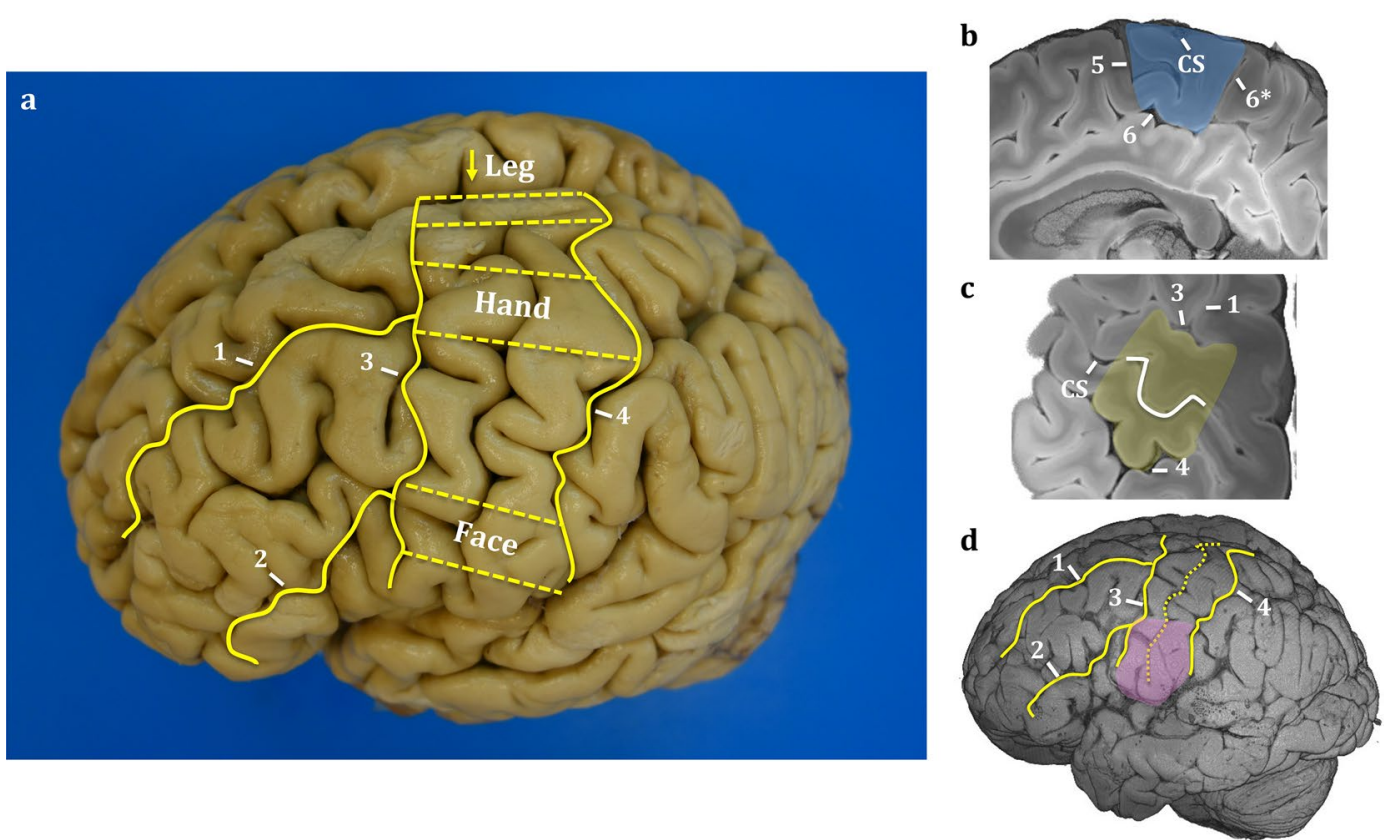

Fig. 2 Motor and somatosensory cortex leg, hand and face region sampling. Identification of motor and somatosensory cortical regions of interest by the sulci (solid yellow lines) on the dorsal and lateral surfaces (a). Dashed yellow lines (a) represents the leg, hand and face regions excised for analysis. Corresponding MR structural images of paracentral lobule (b), hand knob (c) and lateral face (d) areas are highlighted in blue, yellow and pink, respectively. The hand knob recognised by the inverted omega on the central sulcus of the MR structural image (c). Major sulci were used as anatomical landmarks for guided sampling. Annotations - 1: superior frontal sulcus, 2: inferior frontal sulcus, 3: pre-central sulcus, 4: post-central sulcus, 5: paracentral sulcus, 6: cingulate sulcus, 6*: marginal segment of the cingulate sulcus, CS central sulcus, arrow: interhemispheric fissure where leg region sampled on the paracentral lobule

\section{Orbital gyri and gyrus rectus}

The orbital gyri and gyrus rectus, involved in the later stages of ALS (Stage 3) and in the earliest stage of bvFTD (Pattern I), lie in the basal surface of the frontal lobe. The gyrus rectus extends longitudinally in the medial border of the basal surface. The medial orbital gyrus is situated immediately lateral to the gyrus rectus and demarcated by the olfactory sulcus. The gyrus rectus and the medial orbital gyrus were sampled in rostral-caudal axis in serial coronal slices (Fig. 3).

\section{Inferior frontal gyrus (Broca's area)}

Impairment in language and syntactic processing has been implicated in ALS patients [67-70]. Pathological changes in Broca's area, associated with speech production and a wide range of language and communication related functions [71-73], have been reported in ALS with and without other FTD features [67]. Broca's motor speech area and its homologue in the right hemisphere occupy the caudal part of the inferior frontal gyrus demarcated by the precentral sulcus posteriorly, inferior frontal sulcus superiorly and the lateral fissure inferiorly [65]. The area is further delineated macroscopically by the opercular and triangular gyri corresponding to BA
44 and $45[71,74]$. Broca's area was sampled from the inferior frontal gyrus, including the depth of the circular insular sulcus in two coronal slices $(\sim 2 \mathrm{~cm})$ rostral to the precentral sulcus (Fig. 3).

\section{Corticospinal tract, thalamus and striatum}

Identification of the corticospinal tract was guided by diffusion tractography of fibres originating from the leg area of paracentral lobule, and hand and face regions of the precentral gyrus (Fig. 4). The corticospinal projections that descend through the posterior limb of the internal capsule were sampled with the surrounding thalamus and the lentiform nucleus. Following sampling of the corticospinal tract, the remaining basal ganglia and internal capsule were sampled in anterior coronal slices.

\section{Hippocampal formation}

The hippocampal formation of the dorsomedial temporal lobe, involved in the final stage of ALS and representing Pattern II of bvFTD, constitutes the hippocampus with dentate and adjacent cortical regions extending to the parahippocampal gyrus. The parahippocampal gyrus is located inferior to the hippocampus between the hippocampal fissure and collateral sulcus. The hippocampal 

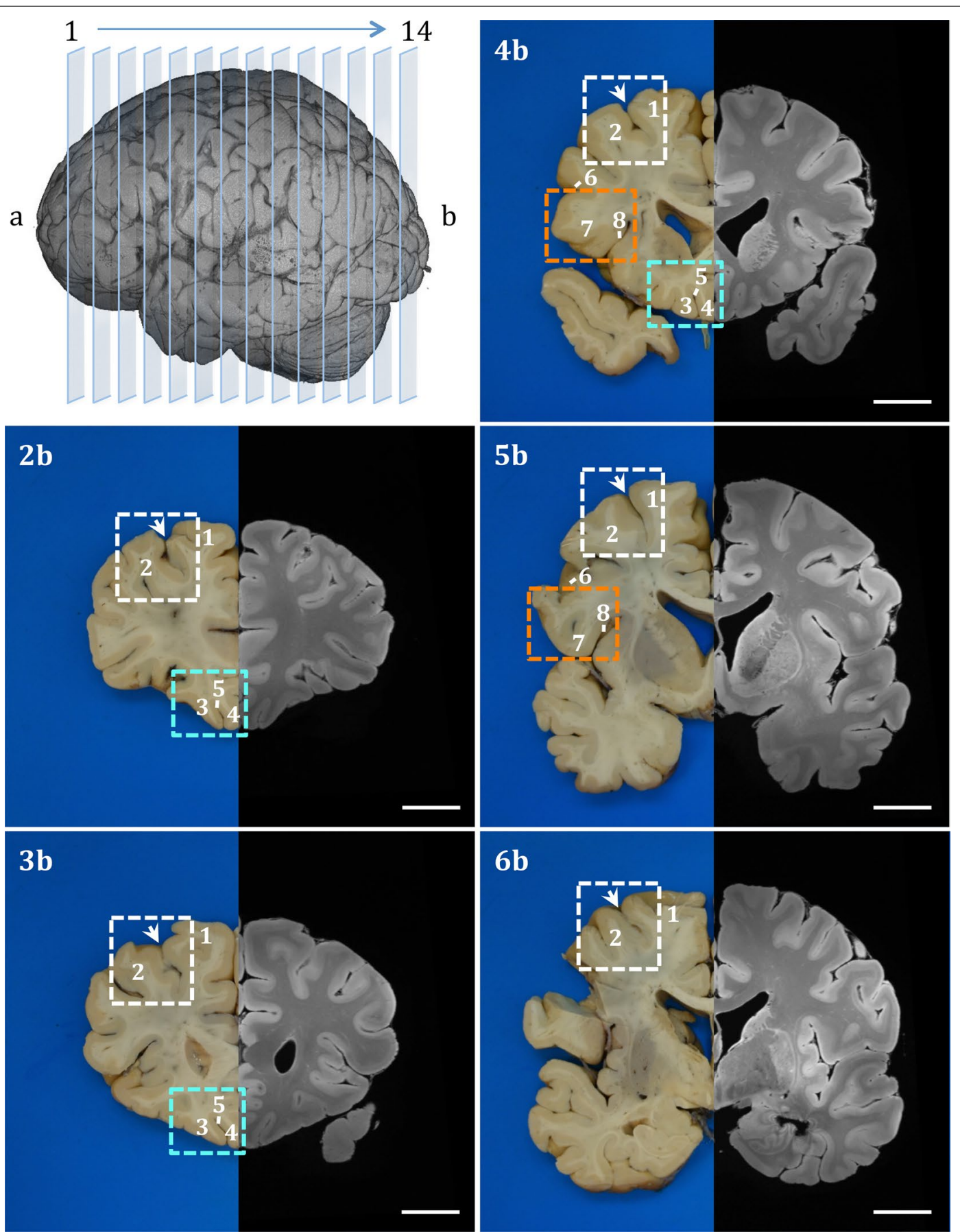

Fig. 3 Systematic sampling strategy for the cortical regions of the frontal lobe. The 3-D MRI image on top left-hand corner represents slicing of the brain on coronal plane. Each slice numbered consecutively in rostral-caudal axis and labelled as anterior (a) or posterior (b) when digitally photographed. Images $\mathbf{2 b}-\mathbf{6 b}$ show gross brain coronal slices (posterior plane) with mirrored structural MR image from the left hemisphere. Key neuroanatomical regions middle frontal gyrus (white rectangle), orbitofrontal cortex (orbital gyri and gyrus rectus: turquoise rectangle) and inferior frontal gyrus (orange rectangle) were sampled in serial coronal slices systematically with their accompanying subcortical white matter. Major sulci were used as landmarks for region and anatomical boundary identification. Annotations-1: superior frontal gyrus, 2: middle frontal gyrus, 3: medial orbital gyrus, 4: gyrus rectus, 5: olfactory sulcus, 6: inferior frontal sulcus, 7: inferior frontal gyrus, 8: circular insular sulcus, arrow: superior frontal sulcus. Scale bar $=2 \mathrm{~cm}$ 


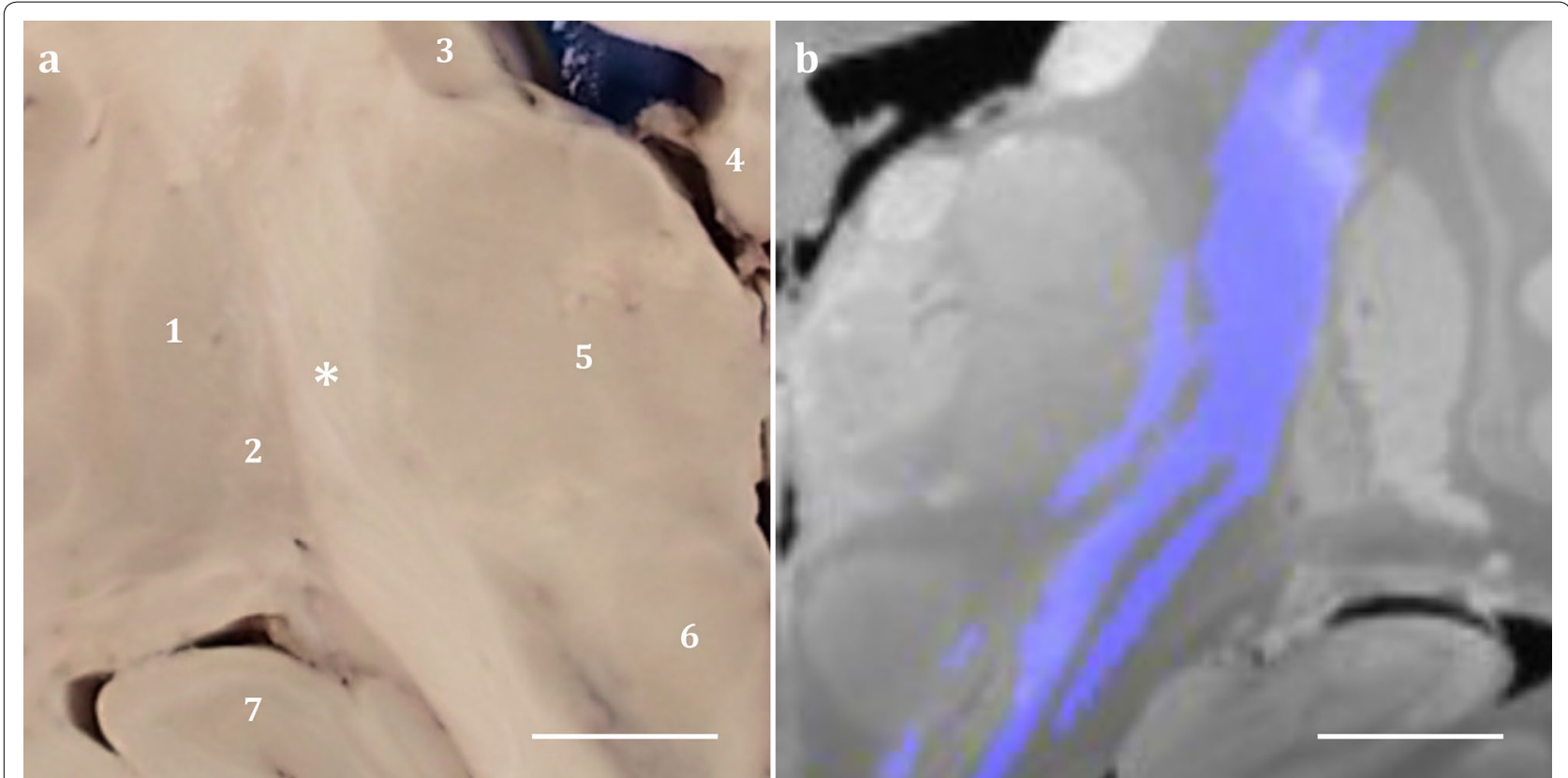

Fig. 4 Tractography guided identification of the corticospinal tract. The slice (a) that best represents the corticospinal tract (purple) was identified with tractography (b mirrored MR image). Annotations-1: putamen, 2: external globus pallidus, 3: caudate, 4: body of fornix, 5: thalamus, 6: red nucleus, 7: hippocampus, * posterior limb of internal capsule. Scale bar $=1 \mathrm{~cm}$

formation, identified by its unique topographical organisation, was sampled in consecutive coronal slices superior to the collateral sulcus.

\section{Corpus callosum, cingulate gyrus and paracallosal cingulum}

The cingulate gyrus is situated on the medial surface of the brain extending from the rostral genu area and dorsally to the body of the corpus callosum and terminating ventral to the splenium [75]. The white matter underlying the cingulate cortex presents the paracallosal cingulum bundle. The cingulate gyrus, inferior to the cingulate sulcus and subparietal sulcus, was sampled serially in the coronal plane together with the corpus callosum.

\section{Fornix}

The fornix, which lies immediately inferior to the corpus callosum, is an arch shaped white matter tract carrying efferent fibres from the hippocampus primarily into the mammillary bodies. The fornix is divided into three regions named crus, body and column along the caudalrostral axis [76]. The crus of the fornix, an extension of the fimbria of the hippocampus, from both hemispheres arches below the splenium of the corpus callosum forming the body of the fornix. The fibre bundle then descends at the rostral end, at the level of the anterior commissure, dividing into the columns of the fornix. Given the shape and compact nature of the fornix, sampling is restricted to macroscopically distinguishable regions such as the body of the fornix (Fig. 4), which can be sampled together with the thalamus or the corpus callosum (refer to previous sections). Presentation of the crus and column of the fornix is dependent on the plane and the level of coronal cut and therefore sampled only when the regions can be macroscopically recognised on the coronal plane.

\section{Brainstem and cerebellum}

The brainstem was sampled perpendicular to its longitudinal axis to include multiple levels of the midbrain, pons and medulla at $5 \mathrm{~mm}$ intervals.

Cerebellar folial pattern and the anatomy of the lobules are best studied in serial parasagittal sections [77, 78]. The cerebellum was bisected sagittally in the midline through the vermis and subsequent slices were cut through the cerebellar hemispheres at 5-7 $\mathrm{mm}$ thickness (Fig. 5). Whole sagittal slices were sampled from vermis to cerebellar hemisphere, including the dentate nucleus.

\section{Internal controls}

In addition to regions that are involved in ALS and associated FTD, regions predicted to have little or no involvement in the disease process were sampled to serve as internal controls. This is a crucial part of the protocol because in our experience any prospective post mortem ultra-high-field MRI study of the human brain is affected 


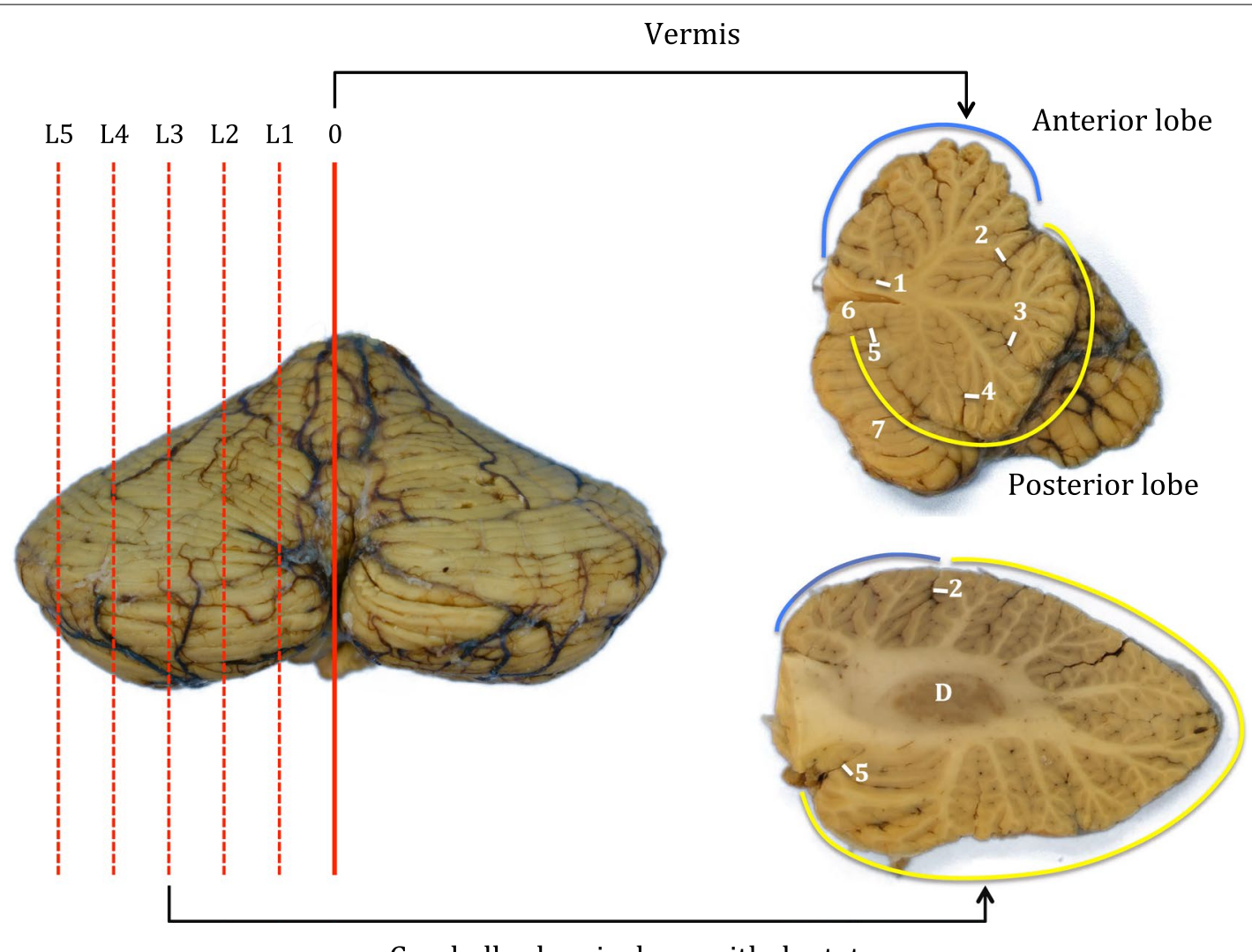

Cerebellar hemisphere with dentate

Fig. 5 Sampling of the cerebellum. Cerebellum sampled on sagittal plane by bisecting through midline of the vermis (level 0) and at subsequent multiple levels (L1-L5) into the cerebellar hemisphere at a set thickness. Annotations-1: precentral fissure, 2: primary fissure, 3: prepyramidal fissure, 4: secondary fissure, 5: posteriolateral fissure, 6: nodulus, 7: tonsil, D dentate, blue line: anterior lobe, yellow line: posterior lobe

by a number of interindividual variables that are difficult to control. However, the strength of a whole brain MRI approach with extensive histological sampling means that an intraindividual calibration of both MRI and histology data is possible via the inclusion of areas that remain unaffected by disease.

The primary visual cortex (BA 17), situated in the medial surface of the occipital lobe, which can be clearly identified by the stria Gennarii at the banks of the calcarine fissure, was sampled with the adjacent secondary visual area (BA 18) and subcortical white matter. The forceps major are radiations of commissural fibres arising from the splenium of the corpus callosum that connects the occipital lobes. Guided by tractography, the forceps major was sampled along the medial wall of the posterior horn of the lateral ventricle and superior to calcar avis on a coronal slice posterior to splenium.

\section{Other regions}

Representative blocks of regions that are not described here were sampled as per standard brain banking protocol for diagnostic purposes.

\section{Histological processing, immunohistochemistry and quantitative digital image analysis}

All sampled tissues were processed for paraffin embedding. Briefly, formalin fixed samples were dehydrated in a series of graded ethanol solutions (70-100\%), cleared in xylene and embedded in paraffin wax. Subsequently, paraffin block surfaces were trimmed and photographed prior to acquiring serial sections at $6-10 \mu \mathrm{m}$ thickness for histology $(10 \mu \mathrm{m}$ thickness for pTDP-43 and $6 \mu \mathrm{m}$ for other stains). Immunohistochemistry was performed with a range of primary antibodies to detect myelin, inflammation (microglia and astroglia activation), iron, neurofilaments and pTDP-43 (Table 3), and visualised using DAKO EnVision Systems and counterstained with haematoxylin. Whole slides were digitised at high resolution ( $\times 40$ objective for pTDP -43 and $\times 20$ objective for all other stains) with Aperio ScanScope ${ }^{\circledR}$ AT Turbo (Leica Biosystems) high-throughput slide scanner.

The relative burden of pathology for immunohistochemical staining in regions of interest was analysed in digital images using Aperio Colour Deconvolution algorithm (version 9.1, Leica Biosystems). Colour 
Table 3 Primary antibodies

\begin{tabular}{|c|c|c|c|c|c|c|}
\hline Antibody & Host & Staining dilution & Supplier & Cat. no. & Remarks & References \\
\hline CD68 (PGM-1) & Mouse & $1: 50$ & DAKO & M0876 & $\begin{array}{l}\text { Marker for activated microglia and mac- } \\
\text { rophages }\end{array}$ & {$[79,81]$} \\
\hline Ferritin & Rabbit & $1: 3000$ & Sigma & F5012 & Iron storage protein & {$[79,83,84]$} \\
\hline GFAP & Rabbit & $1: 2000$ & DAKO & Z0334 & Astroglia marker & {$[79,80]$} \\
\hline lba1 & Rabbit & $1: 1000$ & WAKO & 019-19741 & Microglia marker & {$[81]$} \\
\hline PLP & Mouse & $1: 1000$ & Bio-Rad & MCA839G & Major myelin protein & {$[82]$} \\
\hline pTDP-43 (\$409/410 -clone 9-11) & Mouse & $1: 40000$ & Cosmo Bio & TIP-PTD-M01 & Phosphorylated TDP-43 & {$[27,85,86]$} \\
\hline SMl-311 & Mouse & $1: 1000$ & Biolegend & 837801 & $\begin{array}{l}\text { Pan neuronal marker that recognise non- } \\
\text { phosphorylated epitopes of neurofilament } \\
\text { heavy and medium chains. Stains pyramidal } \\
\text { neurons }\end{array}$ & [87-89] \\
\hline SMI-312 & Mouse & $1: 2000$ & Biolegend & 837901 & $\begin{array}{l}\text { Pan axonal neurofilament marker that recog- } \\
\text { nise phosphorylated epitopes of neurofila- } \\
\text { ment heavy and medium chains }\end{array}$ & {$[87,90]$} \\
\hline
\end{tabular}

deconvolution facilitates stain separation by calibration of colour vectors for each stain (brown immunostain and blue haematoxylin) to generate measurements of selected positive colour channel and intensity thresholding to exclude non-specific background staining. Algorithm input parameters were calibrated and separate threshold for each stain was established by finding a threshold that yielded robust results in at least 10 randomly selected structurally distinct regions [91]. Regions of interests were outlined manually on each image in Aperio ImageScope based on general cytoarchitectural morphology and density differences (Fig. 6), while histological artefacts such as staining artefacts, debris, folds, tears and air bubbles in regions of interest were outlined to exclude from analysis. The colour deconvolution algorithm was applied to quantify the stained area fraction (area of positive staining over total analysis area, Fig. 6) for myelin proteolipid protein (PLP), glial activation (ionised calcium binding adaptor molecule 1: Iba1, cluster of differentiation 68: CD68, glial fibrillary acidic protein: GFAP), ferritin and neurofilaments (SMI-311 and SMI-312), and validated by a neuropathologist. Aperio image analysis algorithms have been used in previous studies to quantify the amount of immunostaining underlying neuropathological changes [92-98] and the fraction of positively stained pixels in an area were reported with demonstrated sensitivity to packing density, size and number of cells and their processes [92, 93, 99].

The colour deconvolution algorithm, as described above, was utilised to quantify the burden of pTDP-43 in manually outlined regions and reported as stained area fraction. Similar algorithms have been used previously for quantification of pathological TDP-43 load [100, 101]. Classification of pTDP-43 phenotype was based on cellular localisation and cortical distribution and categorised into neuronal cytoplasmic inclusions, dystrophic neurites, neuronal intranuclear inclusions, and/or glial inclusions by a neuropathologist according to the accepted criteria [102-104].

\section{Registration}

As illustrated in Fig. 7, the strategy for registration involves several separate stages. All of these are encapsulated in our actively developed software tool (EMMA for Efficient Microscopy-MRI Alignment) [105] that we ultimately intend to release publicly. The first stage in the registration process involves separating the foreground and background for the photographs (Fig. $7 \mathrm{a}-\mathrm{c}$ ). In the second stage the photographed tissue block (or block face) is located within the photograph of the cut-out slice, which we call block face insertion (Fig. 7f). The insertion step itself is a rigid-body registration between the block face (Fig. 7d) and the photograph of the intact brain slice (Fig. 7e). It is informed by the photograph of the cut-out slice (Fig. 7b, after background removal) to minimise the chance of an erroneous insertion. The third stage involves registering the histology images (Fig. $7 \mathrm{~g}$ ) with the block face photograph (Fig. 7d), which are likely to involve distortions and tears associated with slicing sections from the block, but have the same edges. To overcome the resolution gap $(0.5 \mu \mathrm{m} /$ pixel vs. $50 \mu \mathrm{m} /$ pixel $)$, histological images are first sub-sampled to the resolution of the photographs. Tissue shrinkage and large-scale distortions are addressed by an initial affine transformation of the histological images. Finally, small-scale distortions are gradually compensated by smoothly deforming the histological images (Fig. 7g) to follow the edges of the block face (Fig. 7d). To make the registration work across modalities, both the histological images (Fig. 7g) and the block face (Fig. 7d) are represented using the Modality 


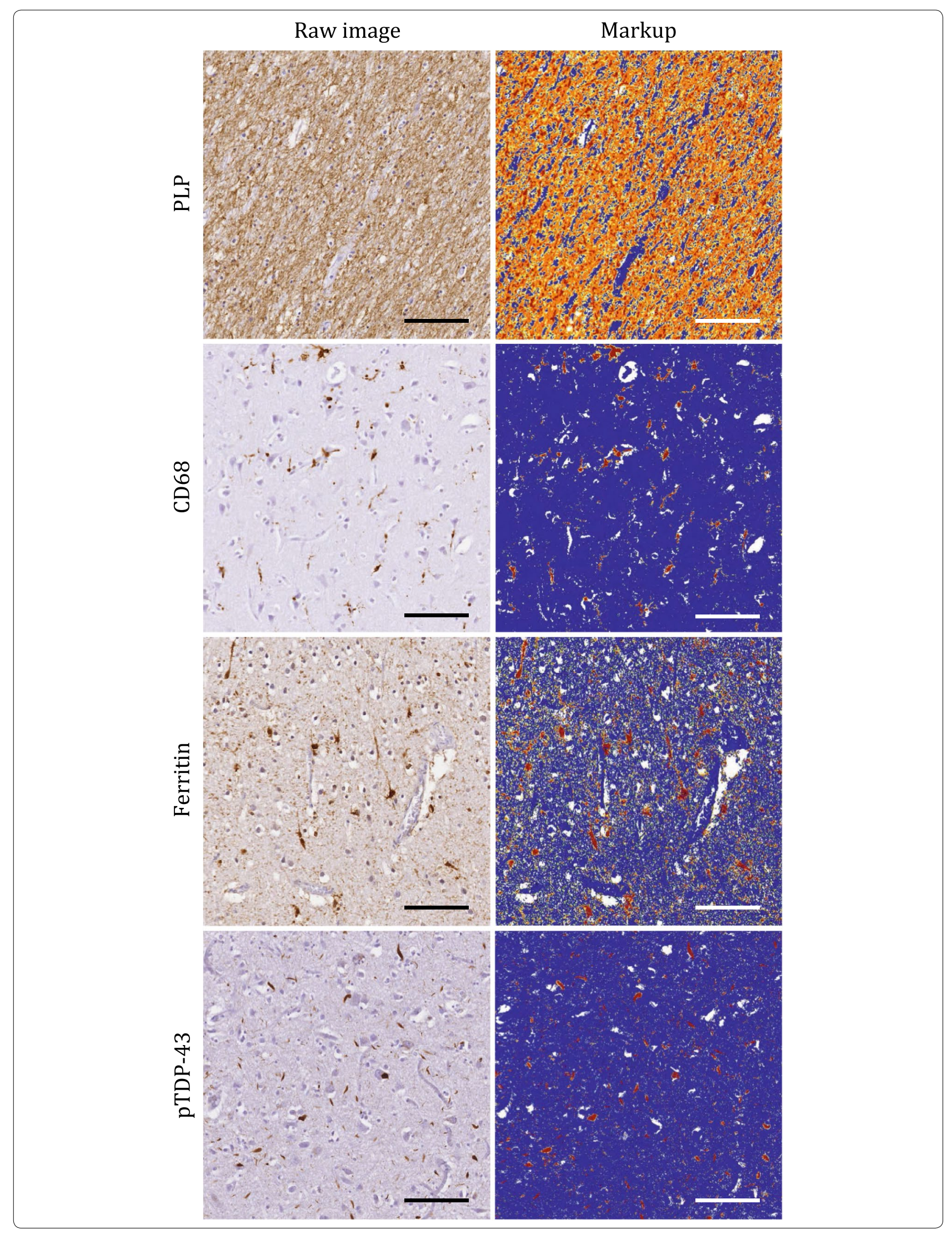


(See figure in previous page)

Fig. 6 Quantitative digital histology image analysis. The high-resolution digital histology images represent the motor cortex subcortical white matter stained for PLP and grey matter stained for CD68, ferritin and PTDP-43 in ALS. Colour deconvolution for positive staining (brown) generates markup images demonstrating unstained pixels $=$ white, negative stained pixels $=$ blue, weak positive pixels $=$ yellow, medium positive pixels $=$ orange and strong positive pixels $=$ red. Scale bar $=100 \mu \mathrm{m}$

Independent Neighbourhood Descriptor (MIND) [106]. MIND was developed for the purpose of cross-modality image registration, and its robustness has already been demonstrated in multiple applications [106], including the registration of brain MR images to histology [107]. The fourth stage involves registering the digital photograph of the intact coronal section (Fig. 7e) to the 3-D MR image of the hemisphere prior to slicing. This registration needs to deal with the relative deformations involved with the brain being placed into the scanner and then cut into slices, meaning that the cut face need not be a simple plane in the 3-D image but would in general be a slightly curved surface. Therefore the MR volume is first re-sampled (Fig. 7h) parallel to a (curvilinear) surface that best represents the anatomical features in the photograph, then grey-white matter boundary information is obtained from a tissue-type segmentation (Fig. 7i to drive the boundary-based registration (BBR) process [108]. The final stage then combines these various stages together, to obtain a non-linear registration of the histology image to the appropriate portion of the 3-D MR image (Fig. 7j). Each stage involves specific challenges but is a lot simpler than a direct registration of the histology image to the 3-D MR image, as a direct registration has far fewer anatomical features to work with as well as very large changes in the type of intensity contrast.

\section{Results}

Preliminary results from each processed MRI modality for a brain are displayed in Fig. 8. The individual modalities display distinctive anatomical contrast and unique information about the underlying tissue composition and microstructure. Furthermore, an example of comparison between multiple histological stains and MRI modalities on a mapped plane from the normal appearing orbitofrontal cortex and its subcortical white matter is demonstrated in Fig. 9. Serial histology sections stained for axonal myelin (PLP) and neurofilament content (SMI-312) were mapped to the corresponding MRI plane (structural and diffusion weighted) that represents the region of interest, spared from pathology, from an ALS patient (ALS 1) (Fig. 9). Qualitative evaluation of the stains demonstrates high myelin and neurofilament stain density in the white matter compared to the grey matter and distinct grey/white matter boundary (Fig. 9). The grey scale structural, fractional anisotropy (FA) and mean diffusivity (MD) images showed comparable contrast in signal intensity that corresponded with histology. Similar observations were made in quantitative FA and MD maps (Fig. 10) indicating sensitivity of these MRI modalities to multiple components of axonal microstructure. Further validation is required with additional stains that represent other microstructural features and should be compared with other MRI modalities for accurate interpretation of these findings.

Proof of concept examining the influence of disease specific microstructural pathological changes on multiple MRI modalities was demonstrated in the post mortem spinal cord from an ALS patient (ALS 2). The MRI acquisition of the spinal cord consisted of a set of protocols (Additional file 1) for structural scan, T1- and T2-mapping, and DW-SSFP. The structural MRI showed lateral corticospinal tract hyperintensity along the scanned segment of the spinal cord (Additional file 2). The latter MRI signal change was concomitant with the degeneration of the lateral corticospinal tract histologically demonstrated with marked axonal myelin and neurofilament loss together with heightened inflammatory response (CD68) on a segment of the cervical cord (Fig. 11 and Table 4). Quantitative evaluation of the same region on the corresponding MRI plane demonstrated significant decrease in average FA value $(P<0.001)$ and significant increase in average MD, T1 and T2 values $(P<0.001)$ in comparison to the normal appearing white matter region (Table 4).

The burden of pathology in the hand knob area of the primary motor cortex grey matter was compared between 2 ALS patients, with varying degree of pathology, and a control (Fig. 12). Qualitative comparisons were made in MRI signal changes in R2* and QSM maps that have been previously demonstrate to be sensitive microglial iron accumulation [7, 9]. Compared to the control, ALS 3 shows moderate diffused hyperintense signal changes in both R2* and susceptibility maps. ALS 4 shows the strongest hyperintense signal change in R2* and susceptibility maps. Upon qualitative and quantitative histological evaluation of the same region, the intensity in signal changes corresponds to the relative burden of CD68 pathology in all cases, with lowest in the control and the highest in ALS 4 (Fig. 12 and Additional file 3). Quantitative pTDP-43 burden appears to be slightly 


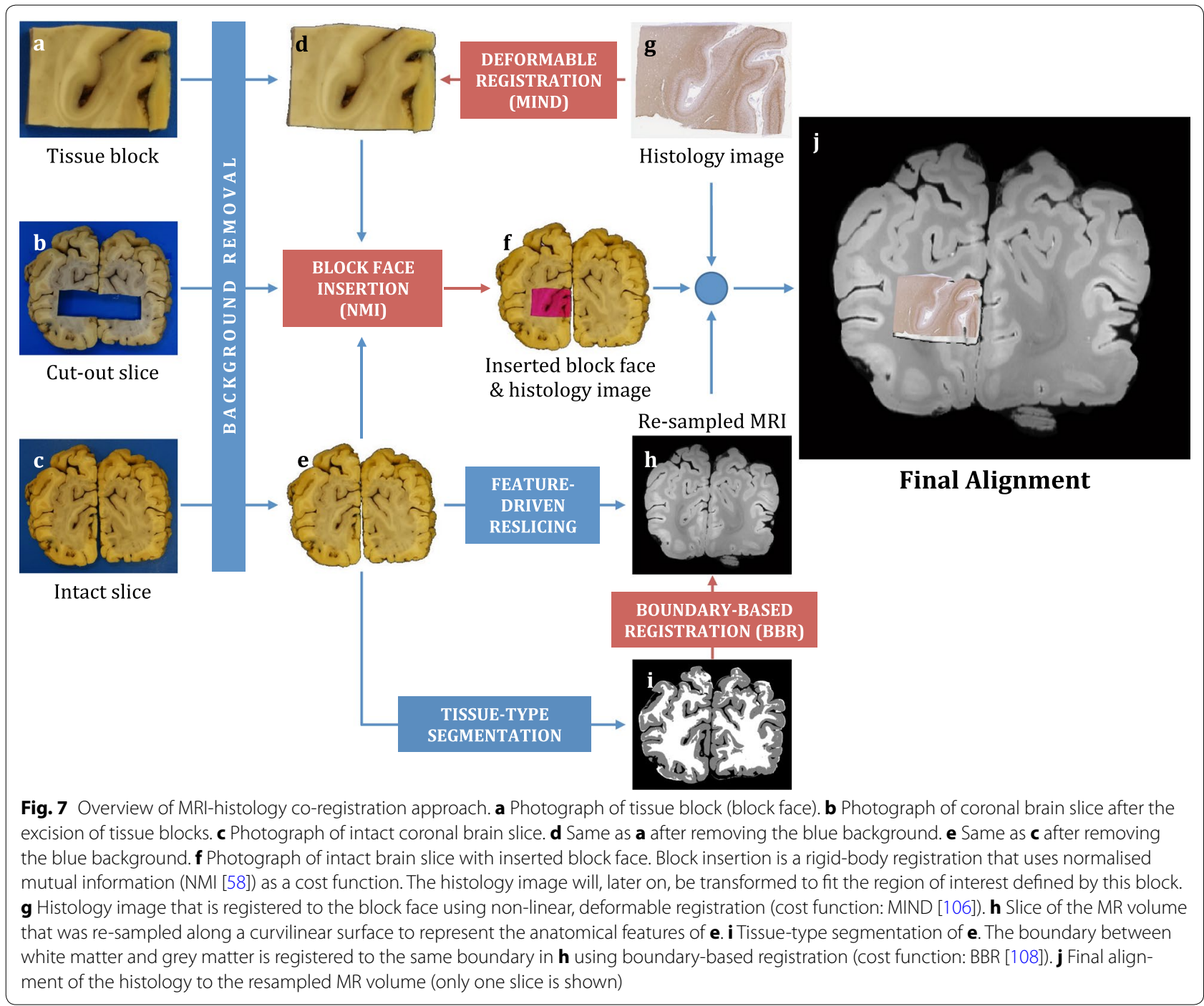

higher in ALS 4 (Additional file 3), however its relationship to CD68 pathology is unclear and further evaluations are warranted. Interestingly, cortical myelin content in the ALS cases was similar to that of the control (Fig. 12 and Additional file 3). Patient data for ALS and control cases reported are presented in Table 5 . The pTDP-43 staging for each ALS case was classified according to the accepted criteria [104].

These findings from the lateral corticospinal tract degeneration of the spinal cord demonstrate multiple MRI modalities that are influenced by several candidate features of microstructural pathological changes. The findings from the motor cortex indicate that this protocol is suitable for the investigation of subtle microstructural changes. However, more robust validation of specificity and sensitivity of these MRI modalities should be extended to a larger cohort and are ideally demonstrated in numerous brain regions with heterogeneous degree of pathological changes. Therefore, the proposed methodology for systematic histological evaluation of pathology propagation in post mortem whole brain in ALS and validating the pathophysiological correlates with MRI signal changes would be ideal to uncover the specificity and sensitivity of MRI measures (Fig. 13).

\section{Discussion}

The extensive landmark based systematic whole brain sampling strategy is aimed for the accurate study of pathological propagation in ALS through identification of regions that represent the proposed four stage pTDP43 spreading pattern with neuroanatomical accuracy. In addition to the sampling of cortical and subcortical grey matter, tractography can be used to guide accurate sampling and histological analysis of white matter tracts of 

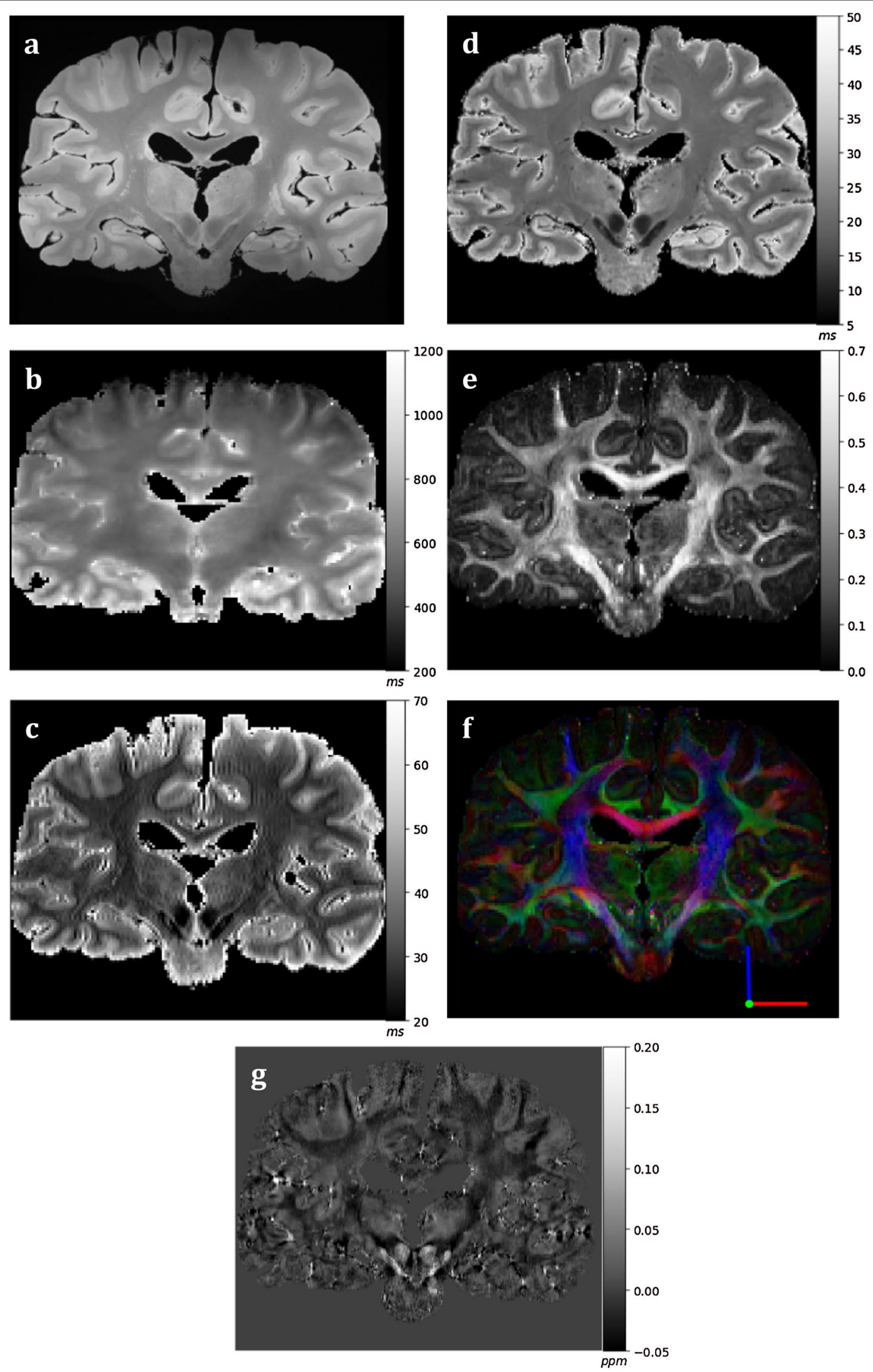
(See figure on previous page)

Fig. 8 Processed MRI datasets from an individual brain. The coronal MR images display a structural map (a), T1-map (b), T2-map (c), T2*-map (d), FA map (e), principal diffusion direction weighted by the FA (f) and susceptibility map ( $(\mathbf{g})$. The calibration bars for the T1-map (b), T2-map (c) and T2*-map (d) indicate the T1,T2 and T2* values (in ms), respectively. The calibration bar in the FA map (e) indicates the fractional anisotropy. The key at bottom right of $\mathbf{f}$ indicates colour orientation of the principal diffusion direction. The calibration bar in the susceptibility map (g) indicates the susceptibility in parts-per-million (ppm)
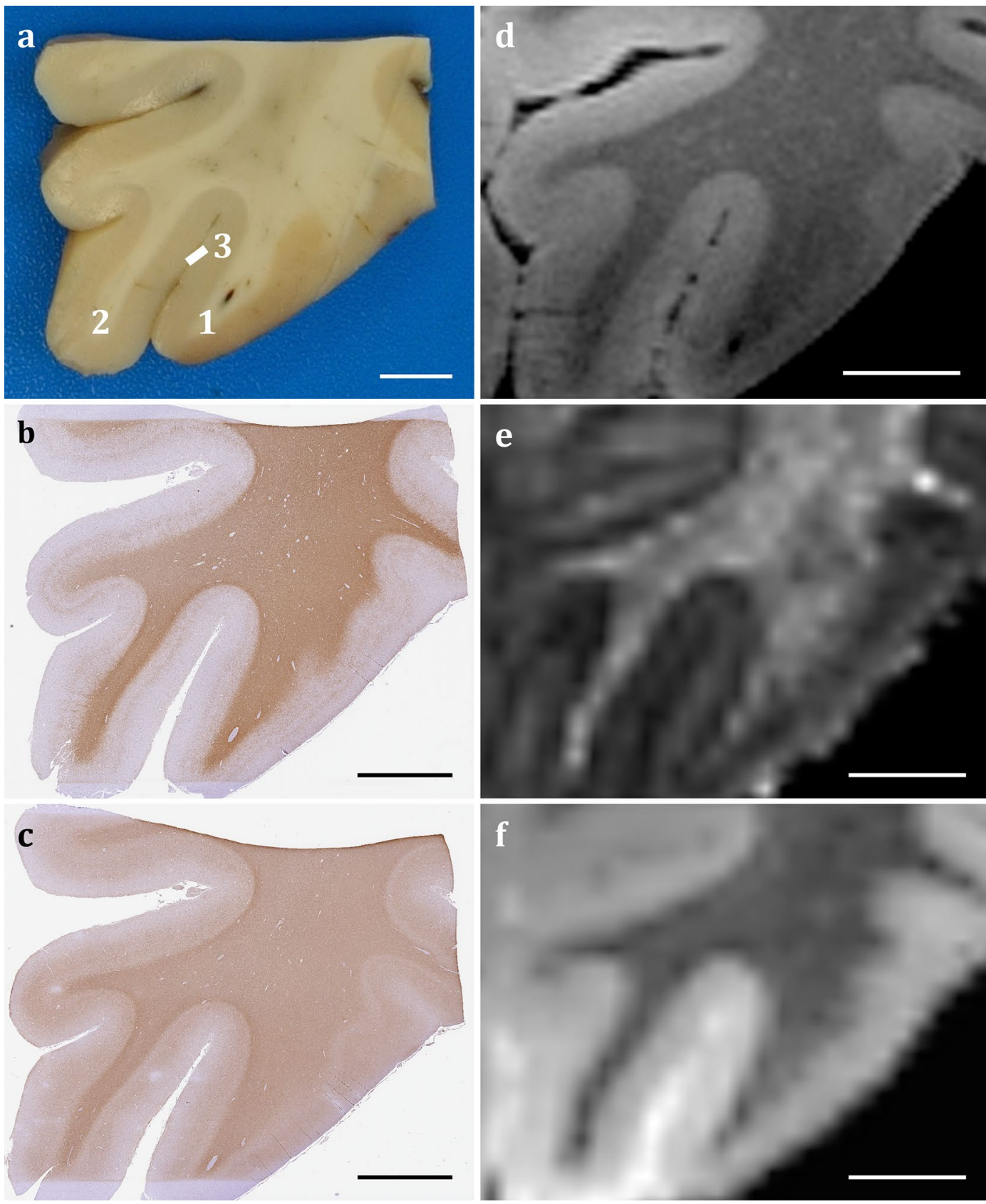

Fig. 9 Example of histology-MRI comparison of the orbitofrontal cortex. Histology sections stained for PLP (b) and SMI-312 (c) were obtained from the block face (a) sampled from the orbitofrontal cortex. High staining intensity of myelin PLP and SMI-312 neurofilament was evident in the subcortical white matter with clear contrast between the cortical grey and white matter boundary. Corresponding plane from structural (d), FA (e) and MD (f) MR images showed changes in signal intensity that were comparable to histology. Annotations-1: medial orbital gyrus, 2: gyrus rectus, 3: olfactory sulcus. Scale bar $=5 \mathrm{~mm}$ 

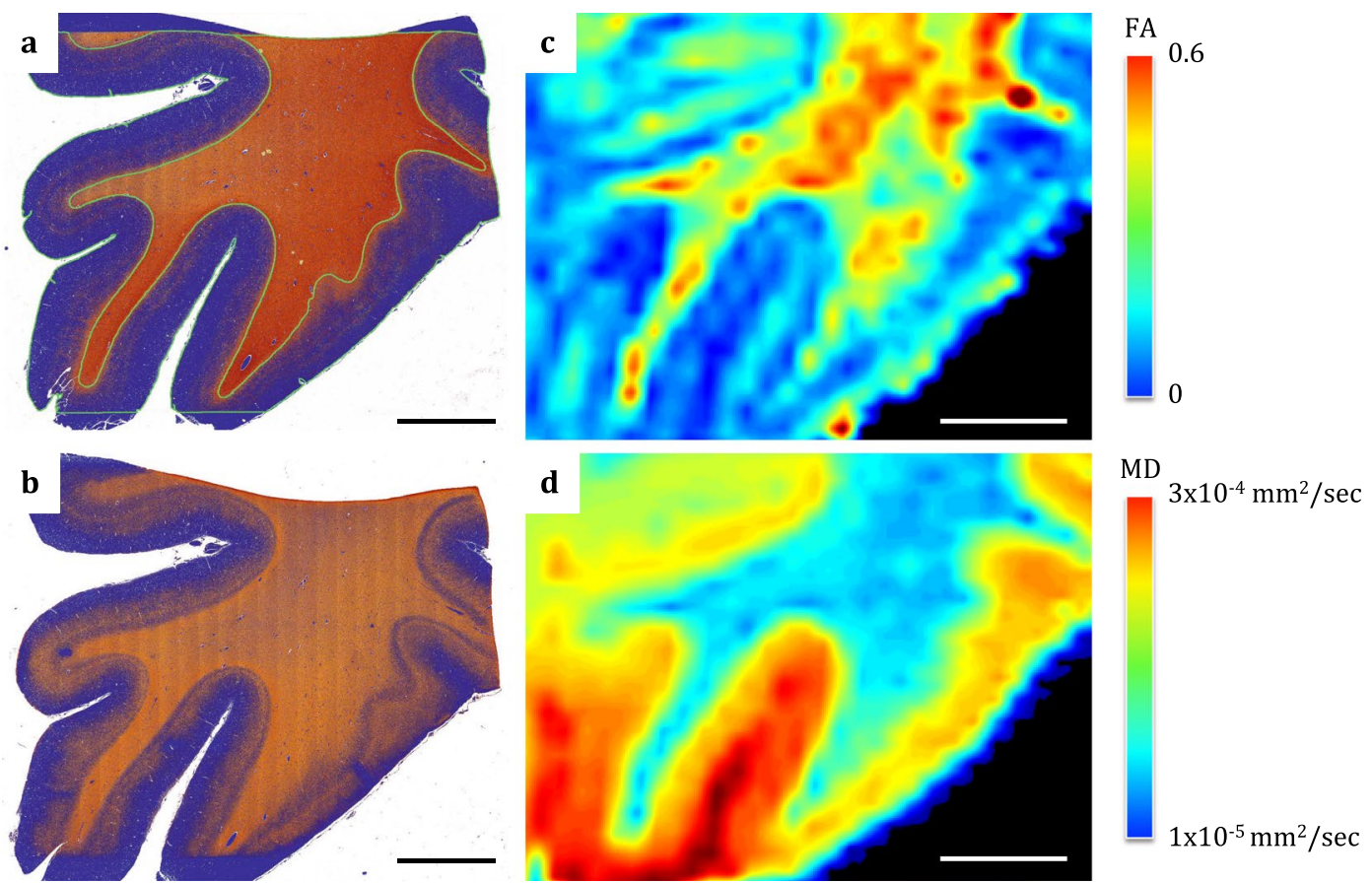

Fig. 10 Histology and MRI quantitative maps. Colour deconvolution markup histology images stained for PLP (a) and SMl-312 (b) demonstrates negative stained pixels = blue, weak positive pixels=yellow, medium positive pixels=orange and strong positive pixels=red. The PLP markup image shows manual outline of the grey matter and the subcortical white matter (green line). Images on the right column demonstrate quantitative FA (c) and MD (d) maps from the corresponding MRI plane and their calibration bars with quantitative values. Scale bar $=5 \mathrm{~mm}$

interest, particularly where these tracts are not demarcated by an anatomical landmark. Systematic sampling of regions of interest was combined with a digital photography pipeline of sampling and histological processing to facilitate alignment and registration required for validation of sensitivity and specificity of MRI modalities to microstructural changes.

One of the attributes of the proposed protocol is sequential sampling of whole regions of interest with distinct topographical features and neuroanatomical landmarks. However, partial sampling was considered for several regions due to size limitations, lack of defined landmarks and adequate representation of pathology spread. Specific examples of the latter include the large middle frontal gyrus, without defined anterior boundary, where sampling was restricted to its dorsal portion immediately lateral to the superior frontal sulcus. In addition, only the main clinicopathologically relevant segments of the primary motor and somatosensory cortex (pre-central and post-central gyri) were sampled. Sampling of the fornix was limited to the body and fimbria, whereas crus and column region extraction on the coronal plane was restricted by slice thickness and variation in cutting angle of coronal slices for macroscopic identification. In such events, accurate MRI-histology correlation in regions of interest may be challenged by the limited availability of histology. Therefore, findings should be interpreted with careful consideration and reported with neuroanatomical accuracy.

Defining white matter tracts on histological images are generally guided by anatomical boundaries derived from location-based histological atlases. Unlike DTI tractography, which facilitates 3-D delineation and visualisation of multiple white matter tracts simultaneously [109], histological delineation of multiple white matter tracts of interest with a common anatomical trajectory on 2-D plane is difficult. For example, the posterior limb of the internal capsule located between the pallidum and thalamus contain corticospinal, corticobulbar and corticopontine projections and thalamic radiations along the superior-inferior axis $[110,111]$. Tractography can be utilised for identification of the histological slice that best depict the tract of interest whilst taking into consideration the other adjacent tracts that may or may not be involved in the disease process. If more than one tract of interest is identified on a single stained histology section, they should be clearly demarcated prior to histological analysis using tractography-derived data. Furthermore, 


\section{a}
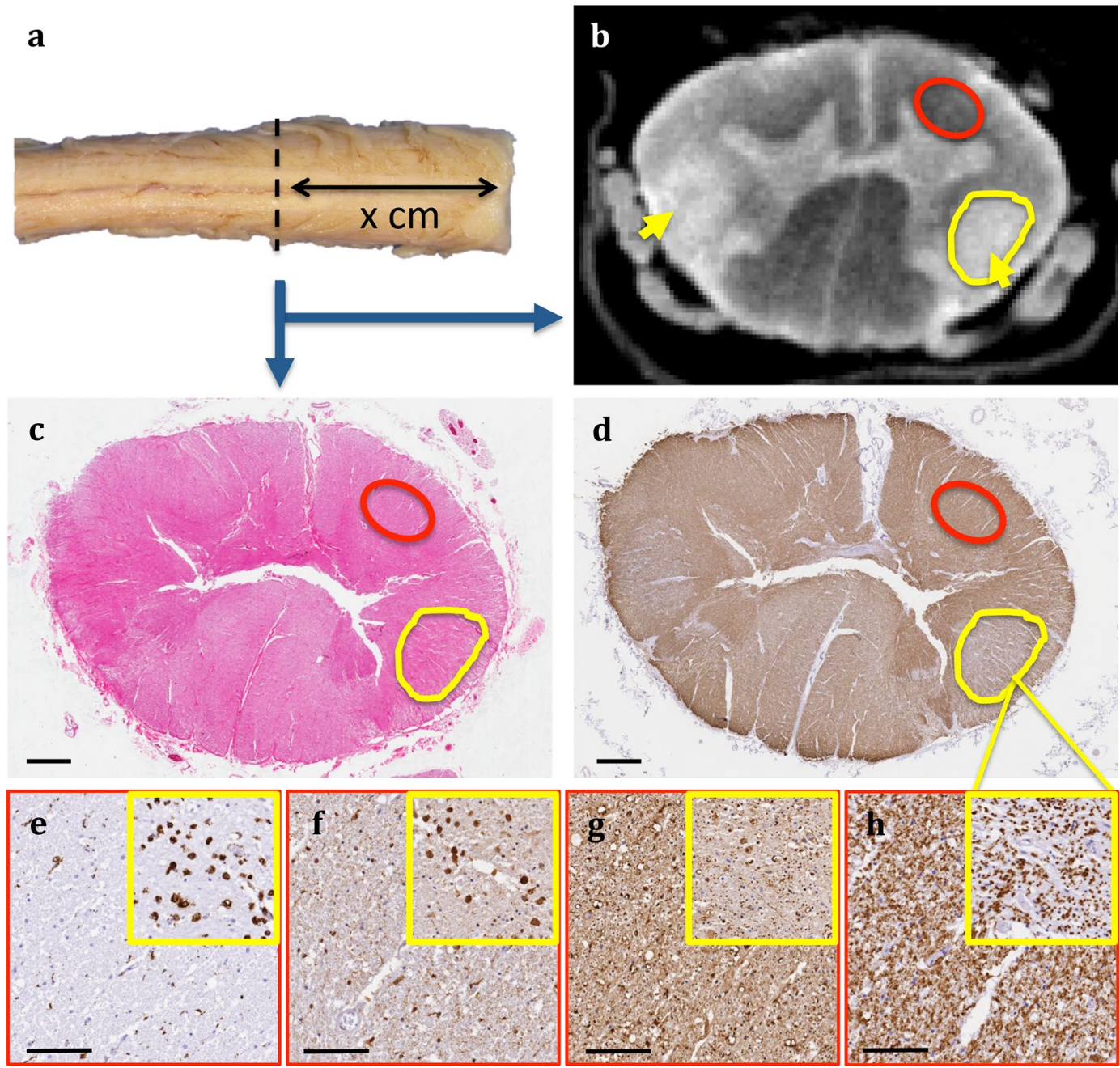

Fig. 11 Qualitative MRI and histological evaluation of the lateral corticospinal tract degeneration in the spinal cord from an ALS patient. MRI and histology was assessed at the same plane (a). The structural MRI (b) shows lateral corticospinal tract hyperintensity (yellow arrow and circle) compared to normal appearing white matter region (red circle). Images $\mathbf{c}$, d represent low magnification snapshots of the spinal cord stained with haematoxylin and eosin and PLP, respectively. Images in the bottom row demonstrate qualitative comparison between normal appearing white matter in red boxes and lateral corticospinal tract in yellow box inserts at $\times 20$ objective magnification for CD68 (e), ferritin (f), SMl-312 (g) and PLP (h) stains. Quantitative MRI and histology analysis outputs for these regions are presented in Table 4. Scale bar for $\mathbf{c}, \mathbf{d}=1 \mathrm{~mm}$. Scale bar for figures e-h and corresponding inserts $=100 \mu \mathrm{m}$

Table 4 Quantitative MRI and histology evaluation of the lateral corticospinal tract of the spinal cord in ALS

\begin{tabular}{|c|c|c|c|c|c|c|c|c|}
\hline \multirow[t]{2}{*}{ Regions } & \multicolumn{4}{|l|}{ MRI } & \multicolumn{4}{|c|}{ Histology } \\
\hline & FA & $\mathrm{MD}\left(\times 10^{-5} \mathrm{~mm}^{2} / \mathrm{s}\right)$ & $\mathrm{T} 1$ (ms) & $\mathrm{T} 2$ (ms) & PLP & SMI-312 & CD68 & Ferritin \\
\hline Lateral Corticospinal Tract & $0.17 \pm 0.03$ & $11.23 \pm 1.19$ & $714.8 \pm 31.4$ & $34.2 \pm 2.11$ & 0.379 & 0.356 & 0.044 & 0.061 \\
\hline Normal appearing white matter & $0.19 \pm 0.07$ & $7.95 \pm 2.52$ & $672.1 \pm 23.6$ & $30.9 \pm 1.31$ & 0.696 & 0.606 & 0.016 & 0.036 \\
\hline$P$ value & $<0.001$ & $<0.001$ & $<0.001$ & $<0.001$ & NA & NA & NA & NA \\
\hline
\end{tabular}

Quantitative MRI and histological data from the lateral corticospinal tract of the spinal cord was compared to the normal appearing white matter region as outlined in Fig. 11. Magnetic resonance imaging data were quantified per voxel in the regions of interest with two-tailed $t$ test and reported as mean \pm standard deviation. The regions of interest within the lateral corticospinal tract and normal appearing white matter were generated from structural MR image and applied to each modality that were analysed. The MR data were calculated in 276 voxels with $100 \mathrm{~mm}^{2}$ cross sectional area in the lateral corticospinal tract and in 269 voxels with 97.6 mm ${ }^{2}$ cross sectional area in the normal appearing white matter. On the corresponding histology plane, a single section was stained for each representative microstructural marker and the stained area fraction (area of positive staining over total analysis area) was calculated 

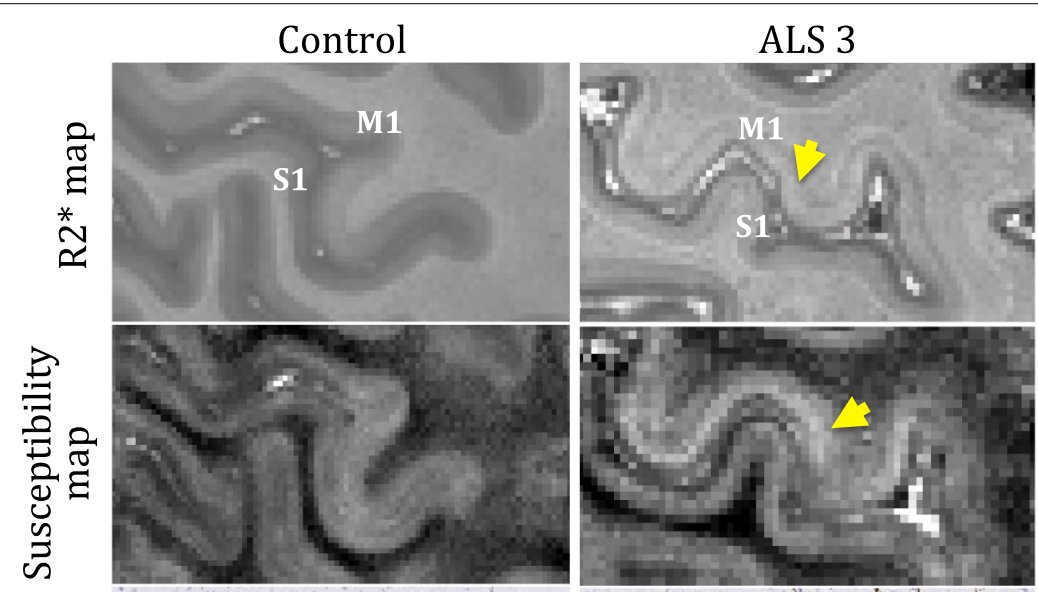

\section{ALS 4}
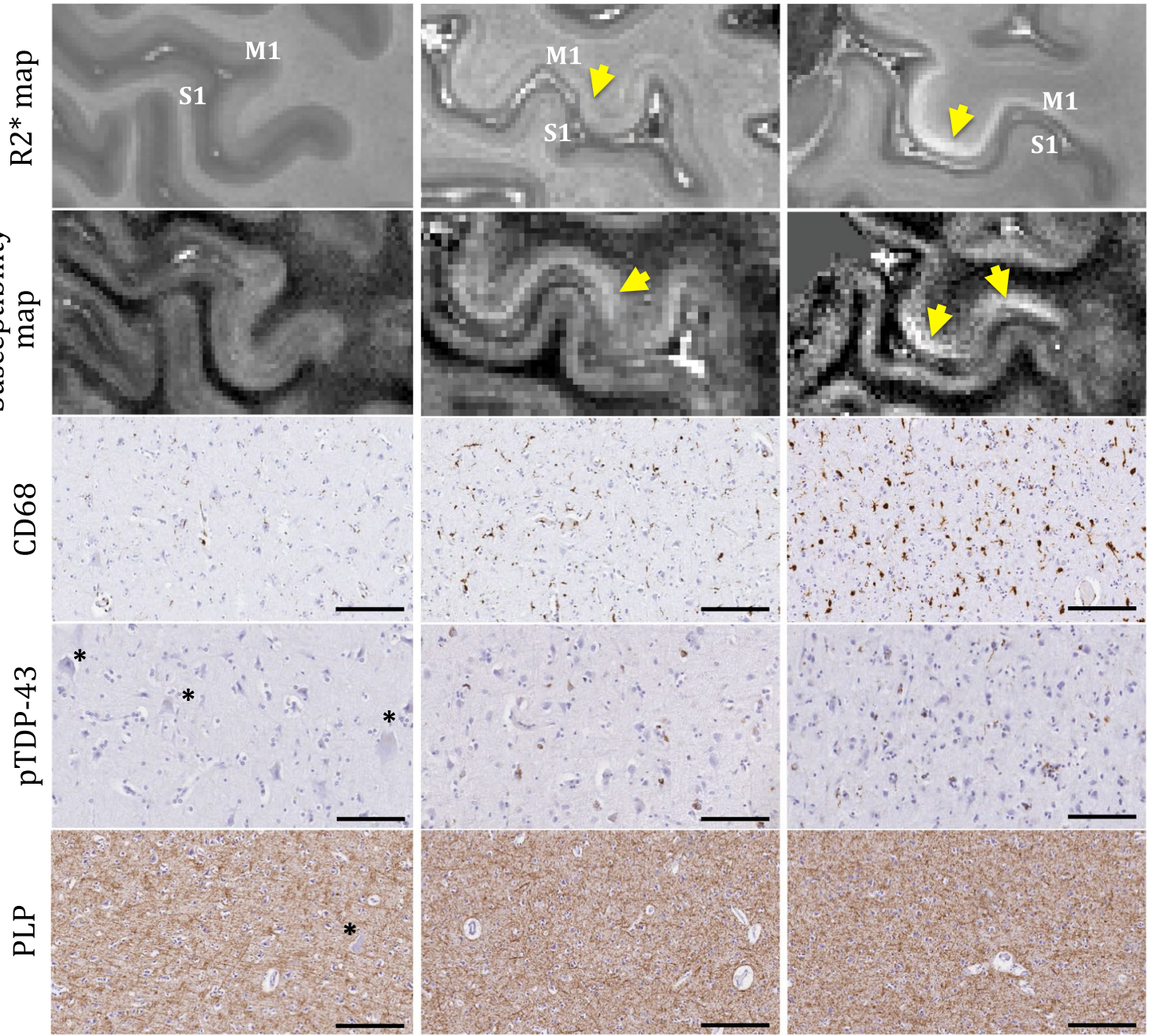

Fig. 12 Qualitative comparison of signal changes in R2* and susceptibility maps and histological evaluation in the primary motor cortex of an age matched control and 2 ALS patients. MRI and histology was assessed in the hand knob region of the primary motor cortex. Evaluations were made specifically in the grey matter at the posterior bank of the precentral gyrus. The first and second row show R2* (inverse of T2*) and susceptibility map in axial plane for a control and 2 ALS patients. Increase in cortical hyperintensity (yellow arrow) is evident in ALS 3 and ALS 4 in comparison to the control. Images in the subsequent rows demonstrate the relative burden of CD68, pTDP-43 and PLP. Quantitative histology analysis outputs are provided in Additional file 3. Annotations-M1: motor cortex, S1: sensory cortex, ${ }^{*}$ Betz cells. Scale bar for CD68 and PLP images $=200 \mu \mathrm{m}$. Scale bar for pTDP-43 images $=100 \mu \mathrm{m}$

interpretation and reporting of findings should consider the possibility of multiple tracts being represented on analytical planes.

The visual cortex and forceps major are regions where ALS associated degenerative changes are not anticipated; they have been used previously as internal controls or reference regions [10, 28, 112]. Conversely, some in vivo imaging studies have reported changes in functional connectivity [113, 114], grey matter atrophy, cortical thinning and altered glucose metabolism in occipital cortex of individuals with ALS independent of visual impairment [115-117]. These findings have not been validated ex vivo, although it has been reported that pTDP-43 pathology may propagate into occipital lobe in the final stages of bvFTD [12]. Growing evidence recognise ALS as a multisystem disorder and given the complex connectivity within the brain, identification of regions that are entirely spared of pathological involvement is challenging. Therefore, identification of internal control regions should be based on the likelihood of no or minimal involvement in the disease process, however their validity should be assessed individually for each 
Table 5 Patient data and neuropathological diagnosis

\begin{tabular}{lllll}
\hline Tissue ID & Age (years) & Sex & $\begin{array}{l}\text { Neuropathologi- } \\
\text { cal evaluation }\end{array}$ & TDP-43 stage \\
\hline Control & 61 & M & Control & - \\
ALS 1 & 68 & M & ALS-TDP type & Stage 4 \\
ALS 2 & 76 & M & ALS with FTD-TDP & Stage 4 and \\
& & & type & Pattern III \\
ALS 3 & 71 & M & ALS-TDP type & Stage 4 \\
ALS 4 & 61 & M & ALS-TDP type & Stage 2 \\
\hline
\end{tabular}

case with histological analysis. It is further essential that molecular and morphological features of the internal control regions are comparable with aged-matched controls with no known neurological disease.

Quantitative characterisation of digital histology images has been recognised as important for advances in high-throughput pathological diagnosis and research, and minimises subjective biases generally expected from traditional qualitative or semi-quantitative (arbitrary rating-scale-based) assessments. Such analytical outputs from histology images provide a comparable platform to correlate against quantitative MR analyses, with the potential to provide an interpretation of MRI-based measures with improved biological specificity. However, the proposed quantitative digital image analysis tool is limited by its inability to discern false positive staining intensity gradient, due methodological challenges, from true positive staining. It is therefore critical to ensure that staining quality is consistently maintained (via batch processing) and all images should be assessed manually to recognise any false positive staining and overall quality prior to analysis.

Accurate alignment of a histology region of interest on the MRI plane is necessary for making direct correlations between the modalities. However, variation in slice plane orientations and spatial resolution in histology and MRI images can have impact on alignment. A typical histology section of $6 \mu \mathrm{m}$ thickness imaged at $\times 20$ objective magnification has an in-plane resolution of $0.502 \mu \mathrm{m} /$ pixel. By comparison, our MR images are of lower resolution with voxel size ranging from $0.25 \mathrm{~mm} \times 0.25 \mathrm{~mm} \times 0.27 \mathrm{~mm}$ for the structural scans to $1.00 \mathrm{~mm} \times 1.00 \mathrm{~mm} \times 1.00 \mathrm{~mm}$ for the T1-and T2-maps. Alignment of smaller regions of interest that are clearly identifiable within a histology image may not be feasible on an MRI plane. Conversely, it is also important to recognise that MRI signal from larger 3-D volume may not always be reflected on a single 2-D histology section [118].

The feasibility of the proposed methodology to better interpret MRI measurements with biological specificity is supported by our preliminary findings in a small number of ALS cases. The lateral corticospinal tract degeneration in the spinal cord of an ALS patient accompanied

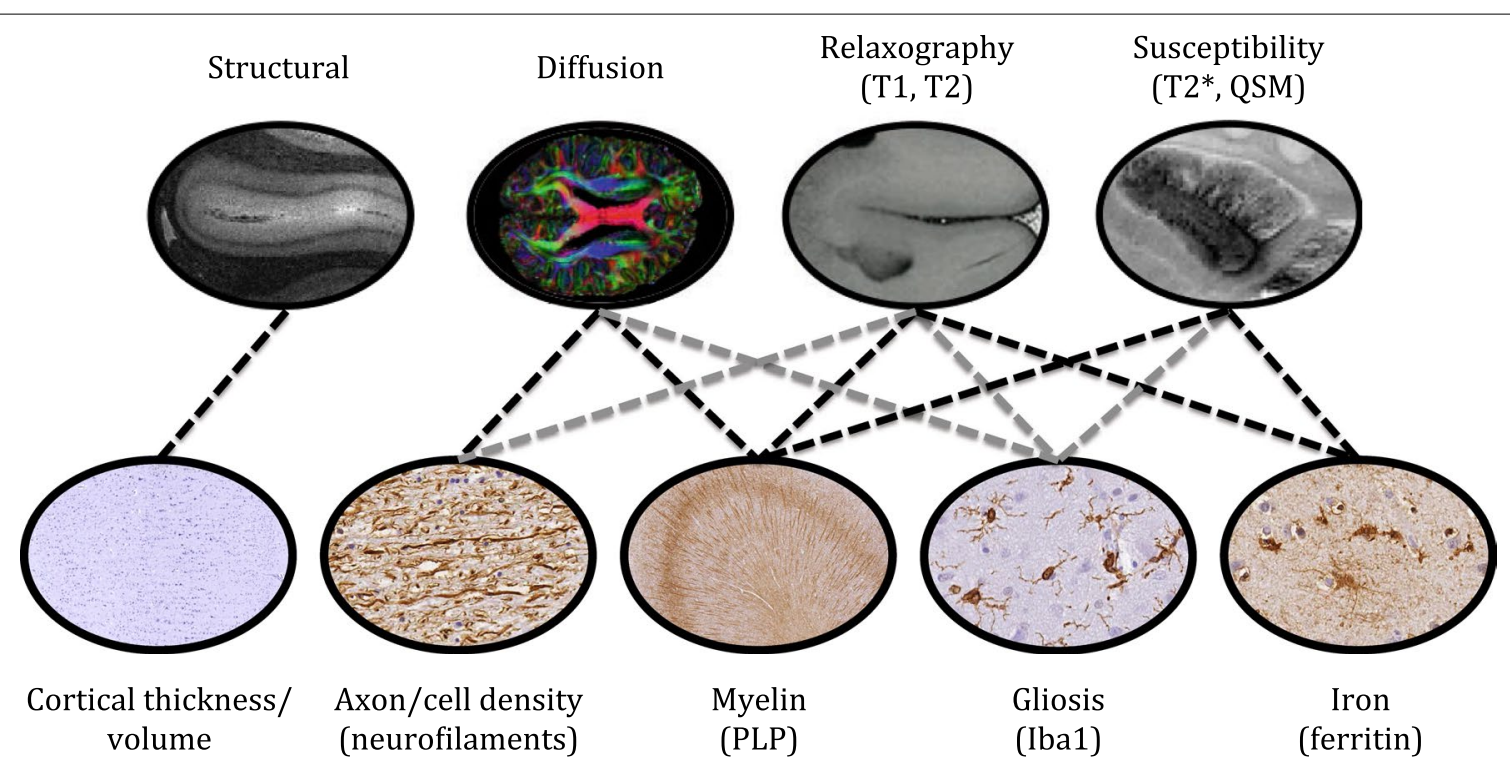

Fig. 13 Predicted sensitivity of MRI modalities to microstructural properties of the neural tissue. Structural, diffusion, relaxography and susceptibility MRI (top row) modalities can be influenced by several aspects of tissue microstructure. The bottom row represents microstructural features and an example of their corresponding histological stain. Predicted relationships between MRI and histology are indicated by dashed lines. The black dashed lines indicate microstructural features that can strongly influence MR signal [7, 107, 119-124]. The grey dashed lines represent other microstructural features that can potentially influence the MR signal (directly or indirectly) and/or to a lesser degree [98, 121]. It should also be noted that any MR measure might show correlation with other microstructural characteristics that are not highlighted in the image 
statistically significant changes in multiple MRI modalities that corresponded with clear axonal degeneration, myelin loss, microglial activation and iron accumulation. Furthermore, the intensity of signal change in R2* and susceptibility maps of the grey matter of the primary motor cortex corresponded primarily with the degree of microglial activation in two ALS cases and a control with comparative changes to pTDP-43 burden. However, the relationship between the CD68 and pTDP-43 expression and their influence (combined and individual) on MR signal is unclear. No difference was observed in myelin content. Previous studies have reported individual microstructural features that can strongly influence a specific MR signal [7, 107, 119-124], however validation of these modalities against multiple molecular and structural features were generally not considered. Recent evidence support the concept that changes to neural microstructure are almost always never isolated to one structural feature and are generally accompanied with several molecular and microstructural changes that can influence the MR signal to a lesser degree $[98,121]$. Therefore, it is the ultimate aim of our project to identify which MRI modality best maps onto which specific neuropathological feature (see Fig. 13). We aim to achieve this through extensive evaluation of multiple regions involved in ALS and FTD pathology spread coupled with histological analysis of multiple microstructural changes that can influence the MR signal in a large cohort.

\section{Conclusion}

The proposed landmark based systematic whole brain sampling strategy of pathologically relevant regions of interest is feasible for routine implementation in a high-throughput manner for the study of disease propagation and direct MRI-histology correlation in ALS. Together with quantitative image analysis and robust registration, this sampling approach facilitates acquisition of large-scale histology datasets for accurate comparisons with quantitative MRI data. This protocol aims to elucidate the relationship of MRI signal changes with underlying pathophysiology. Furthermore, the general principles of this protocol such as identification of regions of interest, systematic sampling, digital photography pipeline for registration and histology image analysis, can be extended to other ex vivo neuroimaging studies with histology correlation.

\section{Additional files}

Additional file 1. Spinal cord MRI protocol parameters.

Additional file 2. Structural MRI from thoracic to cervical region of the spinal cord from an ALS patient.

Additional file 3. Quantitative histological data for the primary motor cortex grey matter at the hand knob.

\section{Abbreviations}

2-D: 2-dimensional; 3-D: 3-dimensional; ALS: amyotrophic lateral sclerosis; BA: Brodmann area; BBR: boundary-based registration; bvFTD: behavioural variant frontotemporal dementia; CD68: cluster of differentiation 68; DTI: diffusion tensor imaging; DW-SSFP: diffusion weighted steady-state free precession; FA: fractional anisotropy; FTD: frontotemporal dementia; GFAP: glial fibrillary acidic protein; Iba1: ionised calcium binding adaptor molecule 1; MD: mean diffusivity; MIND: modality-independent neighbourhood descriptor; MR: magnetic resonance; MRI: magnetic resonance imaging; NMI: normalised mutual information; PLP: proteolipid protein; pTDP-43: phosphorylated 43-kDa TAR DNA-binding protein; SWI: susceptibility weighted imaging; TDP-43: 43-kDa TAR DNA-binding protein.

\section{Author details}

${ }^{1}$ Nuffield Department of Clinical Neurosciences, University of Oxford, Oxford, UK. ${ }^{2}$ Wellcome Centre for Integrative Neuroimaging, FMRIB, Nuffield Department of Clinical Neurosciences, University of Oxford, Oxford, UK. ${ }^{3}$ Department of Radiology, University of Chicago, Chicago, IL, USA.

\section{Authors' contributions}

MPG established the sampling strategy and carried out systematic sampling, block face photography, immunohistochemistry, image analysis, block faceMRI plane alignment and drafted the manuscript. SF established the post mortem MRI protocols carried out MRI acquisition, analysis and drafted the MR imaging segments of the manuscript. RALM carried out tractography and reviewed the manuscript. INH developed block face to MRI plane registration pipeline, generated the registration pipeline image, drafting the registration segment and reviewed the manuscript. MJ contributed to establishment of the block face photography pipeline for registration, developed registration tools and drafted the registration segment of the manuscript. BCT established processing pipelines for MRI modalities and drafted segments of the manuscript. CW carried out processing of the susceptibility-weighted data, generating images and reviewed the manuscript. SJ established analysis tools for MRI processing pipelines and reviewed the manuscript. MRT provided clinical data, supported cohort characterisation and critically appraised the manuscript. KLM established the post mortem MRI protocols and critically appraised the manuscript. OA conceived the study design, contributed to establishment of the sampling strategy and critically appraised the manuscript. All authors read and approved the final manuscript.

\section{Acknowledgements}

We acknowledge the Oxford Brain Bank, supported by the Medical Research Council (MRC), the NIHR Oxford Biomedical Research Centre and the Brains for Dementia Research programme, jointly funded by Alzheimer's Research UK and Alzheimer's Society for supplying the post mortem brains. We also thank the donors and their families.

\section{Competing interests}

The authors declare that they have no competing interests.

\section{Availability of data and materials}

The datasets used and/or analysed to support the conclusion of this article are included in this published article [and its supplementary information files]. Raw data used in the study are available from the corresponding author on reasonable request.

\section{Consent for publication \\ Not applicable.}

Ethics approval and consent to participate

The study was conducted under the Oxford Brain Bank's generic Research Ethics Committee approval (15/SC/0639).

Funding

This study was funded by the grant MR/K02213X/1 from the Medical Research Council (MRC). The Wellcome Trust provided core funding for the Wellcome Centre for Integrative Neuroimaging and fellowship funding for Prof. Miller. Work carried out by Istvan N. Huszar was supported by funding from the Engineering and Physical Sciences Research Council (EPSRC) and Medical Research Council (MRC) [Grant Number EP/L016052/1]. 


\section{Publisher's Note}

Springer Nature remains neutral with regard to jurisdictional claims in published maps and institutional affiliations.

Received: 9 October 2017 Accepted: 2 March 2018

Published online: 13 March 2018

\section{References}

1. Kiernan MC, Vucic S, Cheah BC, Turner MR, Eisen A, Hardiman O, et al. Amyotrophic lateral sclerosis. Lancet. 2011;377:942-55.

2. Burrell JR, Halliday GM, Kril JJ, Ittner LM, Götz J, Kiernan MC, et al. The frontotemporal dementia-motor neuron disease continuum. Lancet. 2016;388:919-31.

3. Turner MR, Talbot K. Mimics and chameleons in motor neurone disease. Pract Neurol. 2013;13:153-64.

4. Turner MR, Verstraete E. What does imaging reveal about the pathology of amyotrophic lateral sclerosis? Curr Neurol Neurosci Rep. 2015;15:569.

5. Menke RAL, Körner S, Filippini N, Douaud G, Knight S, Talbot K, et al. Widespread grey matter pathology dominates the longitudinal cerebral $\mathrm{MRI}$ and clinical landscape of amyotrophic lateral sclerosis. Brain. 2014;137:2546-55.

6. Brady E, Schweitzer A, Liu T, Wang Y, Shahbazi M, Shtilbans A, et al. Quantitative susceptibility mapping (QSM) as a biomarker for C9orf72 familial ALS (P6.027). Neurology. 2015;84:P6-027.

7. Costagli M, Donatelli G, Biagi L, Caldarazzo lenco E, Siciliano G, Tosetti $\mathrm{M}$, et al. Magnetic susceptibility in the deep layers of the primary motor cortex in amyotrophic lateral sclerosis. Neurolmage Clin. 2016;12:965-9.

8. Schweitzer AD, Liu T, Gupta A, Zheng K, Seedial S, Shtilbans A, et al. Quantitative susceptibility mapping of the motor cortex in amyotrophic lateral sclerosis and primary lateral sclerosis. Am J Roentgenol. 2015;204:1086-92.

9. Kwan JY, Jeong SY, Van Gelderen P, Deng H-X, Quezado MM, Danielian LE, et al. Iron accumulation in deep cortical layers accounts for MRI signal abnormalities in ALS: correlating 7 Tesla MRI and pathology. PLoS ONE. 2012;7:e35241.

10. Meadowcroft MD, Mutic NJ, Bigler DC, Wang JL, Simmons Z, Connor $\mathrm{JR}$, et al. Histological-MRI correlation in the primary motor cortex of patients with amyotrophic lateral sclerosis. J Magn Reson Imaging. 2015:41:665-75.

11. Braak H, Brettschneider J, Ludolph AC, Lee VM, Trojanowski JQ, Del Tredici K. Amyotrophic lateral sclerosis-a model of corticofugal axonal spread. Nat Rev Neurol. 2013;9:708-14.

12. Brettschneider J, Del Tredici K, Irwin DJ, Grossman M, Robinson JL, Toledo JB, et al. Sequential distribution of pTDP-43 pathology in behaviorial variant frontotemporal dementia (bvFTD). Acta Neuropathol. 2014;127:423-39.

13. Brettschneider J, Del Tredici K, Lee VMY, Trojanowski JQ. Spreading of pathology in neurodegenerative diseases: a focus on human studies. Nat Rev Neurosci. 2015;16:109-20.

14. Absinta M, Nair G, Filippi M, Ray-Chaudhury A, Reyes-Mantilla MI, Pardo CA, et al. Postmortem magnetic resonance imaging to guide the pathological cut: individualized, 3D-printed cutting boxes for fixed brains. J Neuropathol Exp Neurol. 2014;73:780-8.

15. Annese J. The importance of combining MRI and large-scale digital histology in neuroimaging studies of brain connectivity and disease. Front Neuroinform. 2012:6:13

16. Annese J, Schenker-Ahmed NM, Bartsch H, Maechler P, Sheh C, Thomas $\mathrm{N}$, et al. Postmortem examination of patient H.M.'s brain based on histological sectioning and digital 3D reconstruction. Nat Commun. 2014:5:3122.

17. Ding SL, Royall JJ, Sunkin SM, Ng L, Facer BAC, Lesnar P, et al. Comprehensive cellular-resolution atlas of the adult human brain. J Comp Neurol. 2016:524:3127-481.

18. Mollink J, van Baarsen KM, Dederen PJWC, Foxley S, Miller KL, Jbabdi S, et al. Dentatorubrothalamic tract localization with postmortem MR diffusion tractography compared to histological 3D reconstruction. Brain Struct Funct. 2016;221:3487-501.
19. Kolasinski J, Stagg CJ, Chance SA, DeLuca GC, Esiri MM, Chang E-H, et al. A combined post-mortem magnetic resonance imaging and quantitative histological study of multiple sclerosis pathology. Brain. 2012;135:2938-51.

20. Ravits J, Paul P, Jorg C. Focality of upper and lower motor neuron degeneration at the clinical onset of ALS. Neurology. 2007;68:1571-5.

21. Ravits J, Appel S, Baloh RH, Barohn R, Brooks BR, Elman L, et al. Deciphering amyotrophic lateral sclerosis: what phenotype, neuropathology and genetics are telling us about pathogenesis. Amyotrop Lateral Scler Frontotemporal Degener. 2013;14:5-18.

22. Neumann M, Mackenzie IR, Cairns NJ, Boyer PJ, Markesbery WR, Smith CD, et al. TDP-43 in the ubiquitin pathology of frontotemporal dementia with VCP gene mutations. J Neuropathol Exp Neurol. 2007;66:152-7.

23. Neumann M, Sampathu DM, Kwong LK, Truax AC, Micsenyi MC, Chou TT, et al. Ubiquitinated TDP-43 in frontotemporal lobar degeneration and amyotrophic lateral sclerosis. Science. 2006:314:130-3.

24. Brettschneider J, Arai K, Del Tredici K, Toledo J, Robinson J, Lee E, et al. TDP-43 pathology and neuronal loss in amyotrophic lateral sclerosis spinal cord. Acta Neuropathol. 2014;128:423-37.

25. Brettschneider J, Del Tredici K, Toledo JB, Robinson JL, Irwin DJ, Grossman M, et al. Stages of pTDP-43 pathology in amyotrophic lateral sclerosis. Ann Neurol. 2013:74:20-38.

26. Pressman PS, Miller BL. Diagnosis and management of behaviorial variant frontotemporal dementia. Biol Psychiatry. 2014;75:574-81.

27. Fatima M, Tan R, Halliday GM, Kril JJ. Spread of pathology in amyotrophic lateral sclerosis: assessment of phosphorylated TDP-43 along axonal pathways. Acta Neuropathol Commun. 2015:3:47.

28. Kassubek J, Müller H-P, Del Tredici K, Brettschneider J, Pinkhardt EH, Lulé $D$, et al. Diffusion tensor imaging analysis of sequential spreading of disease in amyotrophic lateral sclerosis confirms patterns of TDP-43 pathology. Brain. 2014;137:1733-40.

29. Hornberger M, Geng J, Hodges JR. Convergent grey and white matter evidence of orbitofrontal cortex changes related to disinhibition in behavioural variant frontotemporal dementia. Brain. 2011;134:2502-12.

30. Mahoney CJ, Ridgway GR, Malone IB, Downey LE, Beck J, Kinnunen KM, et al. Profiles of white matter tract pathology in frontotemporal dementia. Hum Brain Mapp. 2014:35:4163-79.

31. Tovar-Moll F, de Oliveira-Souza R, Bramati IE, Zahn R, Cavanagh A, Tierney $\mathrm{M}$, et al. White matter tract damage in the behavioral variant of frontotemporal and corticobasal dementia syndromes. PLoS ONE. 2014;9:e102656.

32. Hornberger $\mathrm{M}$, Wong $\mathrm{S}$, Tan $\mathrm{R}$, Irish $\mathrm{M}$, Piguet $\mathrm{O}$, Kril J, et al. In vivo and post-mortem memory circuit integrity in frontotemporal dementia and Alzheimer's disease. Brain. 2012:135:3015-25.

33. Takahashi T, Yagishita S, Amano N, Yamaoka K, Kamei T. Amyotrophic lateral sclerosis with numerous axonal spheroids in the corticospinal tract and massive degeneration of the cortex. Acta Neuropathol. 1997;94:294-9.

34. Sugiyama M, Takao M, Hatsuta H, Funabe S, Ito S, Obi T, et al. Increased number of astrocytes and macrophages/microglial cells in the corpus callosum in amyotrophic lateral sclerosis. Neuropathology. 2013;33:591-9.

35. Brettschneider TJB, Van Deerlin VM, Elman L, McCluskey L, Lee VMY, et al. Microglial activation correlates with disease progression and upper motor neuron clinical symptoms in amyotrophic lateral sclerosis. PLoS ONE. 2012;7:e39216.

36. Turner MR, Menke RA. The tough body at the epicentre of amyotrophic lateral sclerosis. J Neurol Neurosurg Psychiatry. 2017;88:369-70.

37. Filippini N, Douaud G, Mackay CE, Knight S, Talbot K, Turner MR. Corpus callosum involvement is a consistent feature of amyotrophic lateral sclerosis. Neurology. 2010;75:1645-52.

38. Muller HP, Turner MR, Grosskreutz J, Abrahams S, Bede P, Govind V, et al. A large-scale multicentre cerebral diffusion tensor imaging study in amyotrophic lateral sclerosis. J Neurol Neurosurg Psychiatry. 2016;87:570-9.

39. Geser F, Brandmeir NJ, Kwong LK, et al. Evidence of multisystem disorder in whole-brain map of pathological TDP-43 in amyotrophic lateral sclerosis. Arch Neurol. 2008;65:636-41.

40. Amunts K, Lepage C, Borgeat L, Mohlberg H, Dickscheid T, Rousseau M-É, et al. BigBrain: an ultrahigh-resolution 3D human brain model. Science. 2013;340:1472-5. 
41. Perl DP, Good PF, Bussière T, Morrison JH, Erwin JM, Hof PR. Practical approaches to stereology in the setting of aging- and disease-related brain banks. J Chem Neuroanat. 2000;20:7-19.

42. Ou Y, Shen D, Feldman M, Tomaszewski J, Davatzikos C. Non-rigid registration between histological and MR images of the prostate: a joint segmentation and registration framework. In: IEEE computer society conference on computer vision and pattern recognition workshops; 2009, pp 125-32.

43. Alegro M, Amaro-Jr E, Loring B, Heinsen H, Alho E, Zollei L, et al. Multimodal whole brain registration: MRI and high resolution histology. In: Proceedings of the IEEE conference on computer vision and pattern recognition workshops; 2016, pp 194-202

44. Dauguet J, Delzescaux T, Condé F, Mangin J-F, Ayache N, Hantraye P, et al. Three-dimensional reconstruction of stained histological slices and $3 \mathrm{D}$ non-linear registration with in vivo MRI for whole baboon brain. J Neurosci Methods. 2007;164:191-204.

45. Malandain G, Bardinet É, Nelissen K, Vanduffel W. Fusion of autoradiographs with an MR volume using 2-D and 3-D linear transformations. Neuroimage. 2004;23:111-27.

46. Schormann T, Zilles K. Three-dimensional linear and nonlinear transformations: an integration of light microscopical and MRI data. Hum Brain Mapp. 1998:6:339-47.

47. Mega MS, Chen SS, Thompson PM, Woods RP, Karaca TJ, Tiwari A, et al. Mapping histology to metabolism: coregistration of stained wholebrain sections to premortem PET in Alzheimer's disease. Neuroimage. 1997;5:147-53.

48. Foxley S, Jbabdi S, Clare S, Lam W, Ansorge O, Douaud G, et al. Improving diffusion-weighted imaging of post-mortem human brains: SSFP at 7T. Neuroimage. 2014;102(Pt 2):579-89.

49. Miller KL, McNab JA, Jbabdi S, Douaud G. Diffusion tractography of post-mortem human brains: optimization and comparison of spin echo and steady-state free precession techniques. Neuroimage. 2012:59:2284-97.

50. McNab JA, Jbabdi S, Deoni SC, Douaud G, Behrens TE, Miller KL. High resolution diffusion-weighted imaging in fixed human brain using diffusion-weighted steady state free precession. Neuroimage. 2009;46:775-85.

51. Jones E, Oliphant T, Peterson P. SciPy: open source scientific tools for python. 2001. http://www.scipy.org/. Accessed 20 Sept 2017.

52. Svd Walt, Colbert SC, Varoquaux G. The NumPy array: a structure for efficient numerical computation. Comput Sci Eng. 2011;13:22-30.

53. Behrens TE, Berg HJ, Jbabdi S, Rushworth MF, Woolrich MW. Probabilistic diffusion tractography with multiple fibre orientations: What can we gain? Neuroimage. 2007;34:144-55.

54. Jenkinson M, Beckmann CF, Behrens TE, Woolrich MW, Smith SM. FSL. Neuroimage. 2012;62:782-90.

55. Hernández M, Guerrero GD, Cecilia JM, García JM, Inuggi A, Jbabdi S, et al. Accelerating fibre orientation estimation from diffusion weighted magnetic resonance imaging using GPUs. PLoS ONE. 2013;8:e61892.

56. McNab JA, Miller KL. Steady-state diffusion-weighted imaging: theory, acquisition and analysis. NMR Biomed. 2010;23:781-93.

57. Jenkinson M, Bannister P, Brady M, Smith S. Improved optimization for the robust and accurate linear registration and motion correction of brain images. Neuroimage. 2002;17:825-41.

58. Jenkinson M, Smith S. A global optimisation method for robust affine registration of brain images. Med Image Anal. 2001;5:143-56.

59. Schofield MA, Zhu Y. Fast phase unwrapping algorithm for interferometric applications. Opt Lett. 2003;28:1194-6.

60. Özbay PS, Deistung A, Feng X, Nanz D, Reichenbach JR, Schweser F. A comprehensive numerical analysis of background phase correction with V-SHARP. NMR Biomed. 2017;30(4):e3550. https://doi.org/10.1002/ nbm. 3550

61. Wei H, Dibb R, Zhou Y, Sun Y, Xu J, Wang N, et al. Streaking artifact reduction for quantitative susceptibility mapping of sources with large dynamic range. NMR Biomed. 2015;28:1294-303.

62. Pinkhardt EH, Jurgens $\mathrm{R}$, Becker W, Molle M, Born J, Ludolph AC, et al. Signs of impaired selective attention in patients with amyotrophic lateral sclerosis. J Neurol. 2008;255:532-8.

63. Seer C, Furkotter S, Vogts MB, Lange F, Abdulla S, Dengler R, et al. Executive dysfunctions and event-related brain potentials in patients with amyotrophic lateral sclerosis. Front Aging Neurosci. 2015;7:225.
64. Vellage AK, Veit M, Kobeleva X, Petri S, Vielhaber S, Muller NG. Working memory network changes in ALS: an fMRI study. Front Neurosci. 2016;10:158.

65. Petrides M, Pandya DN. Chapter 25-the frontal cortex A2. In: Paxinos G, Mai JK, editors. The human nervous system. 2nd ed. San Diego: Academic Press; 2004. p. 950-72.

66. Petrides M, Pandya DN. Dorsolateral prefrontal cortex: comparative cytoarchitectonic analysis in the human and the macaque brain and corticocortical connection patterns. Eur J Neurosci. 1999:11:1011-36.

67. BakTH, O'Donovan DG, Xuereb JH, Boniface S, Hodges JR. Selective impairment of verb processing associated with pathological changes in Brodmann areas 44 and 45 in the motor neurone disease-dementiaaphasia syndrome. Brain. 2001;124:103-20.

68. Yoshizawa K, Yasuda N, Fukuda M, Yukimoto Y, Ogino M, Hata W, et al. Syntactic comprehension in patients with amyotrophic lateral sclerosis. Behav Neurol. 2014;2014:230578. https://doi.org/10.1155/2014/230578.

69. Tsermentseli S, Leigh PN, Taylor LJ, Radunovic A, Catani M, Goldstein LH. Syntactic processing as a marker for cognitive impairment in amyotrophic lateral sclerosis. Amyotrop Lateral Scler Frontotemporal Degener. 2016;17:69-76.

70. BakTH, Hodges JR. The effects of motor neurone disease on language: further evidence. Brain Lang. 2004;89:354-61.

71. Nishitani N, Schürmann M, Amunts K, Hari R. Broca's region: from action to language. Physiology. 2005;20:60-9.

72. Embick D, Marantz A, Miyashita Y, O’Neil W, Sakai KL. A syntactic specialization for Broca's area. Proc Natil Acad Sci USA. 2000;97:6150-4.

73. Fadiga L, Craighero L. Hand actions and speech representation in Broca's area. Cortex. 2006;42:486-90.

74. Zilles K. Chapter 27-architecture of the human cerebral cortex: regional and laminar organization A2. In: Paxinos G, Mai JK, editors. The human nervous system. 2nd ed. San Diego: Academic Press; 2004. p. 997-1055.

75. Vogt BA, Vogt $L$, Hof PR. Chapter 24 - Cingulate gyrus A2. In: Paxinos G, Mai JK, editors. The human nervous system. 2nd ed. San Diego: Academic Press; 2004. p. 915-49.

76. Mark LP, Daniels DL, Naidich TP. The fornix. AJNR Am J Neuroradiol. 1993;14:1355-8.

77. Press GA, Murakami JW, Courchesne E, Grafe M, Hesselink JR. The cerebellum: 3. anatomic-MR correlation in the coronal plane. AJR Am J Roentgenol. 1990;154:593-602.

78. Press GA, Murakami J, Courchesne E, Berthoty DP, Grafe M, Wiley CA, et al. The cerebellum in sagittal plane-anatomic-MR correlation: 2 . The cerebellar hemispheres. AJR Am J Roentgenol. 1989;153:837-46.

79. Bagnato F, Hametner S, Yao B, van Gelderen P, Merkle H, Cantor FK, et al. Tracking iron in multiple sclerosis: a combined imaging and histopathological study at 7 Tesla. Brain. 2011:134:3602-15.

80. Eng LF. Glial fibrillary acidic protein (GFAP): the major protein of glial intermediate filaments in differentiated astrocytes. J Neuroimmunol. 1985:8:203-14.

81. Korzhevskii DE, Kirik OV. Brain microglia and microglial markers. Neurosci Behav Physiol. 2016:46:284-90.

82. Nave K-A, Werner HB. Myelination of the nervous system: mechanisms and functions. Annu Rev Cell Dev Biol. 2014:30:503-33.

83. Rouault TA. Iron metabolism in the CNS: implications for neurodegenerative diseases. Nat Rev Neurosci. 2013:14:551-64.

84. van Duijn S, Nabuurs RJA, van Duinen SG, Natté R. Comparison of histological techniques to visualize iron in paraffin-embedded brain tissue of patients with Alzheimer's disease. J Histochem Cytochem. 2013;61:785-92.

85. Tan R, Shepherd C, Kril J, McCann H, McGeachie A, McGinley C, et al. Classification of FTLD-TDP cases into pathological subtypes using antibodies against phosphorylated and non-phosphorylated TDP43. Acta Neuropathol Commun. 2013;1:33

86. Takeuchi R, Tada M, Shiga A, Toyoshima Y, Konno T, Sato T, et al. Heterogeneity of cerebral TDP-43 pathology in sporadic amyotrophic lateral sclerosis: evidence for clinico-pathologic subtypes. Acta Neuropathol Commun. 2016;4:61.

87. Ulfig N, Nickel J, Bohl J. Monoclonal antibodies SMI 311 and SMI 312 as tools to investigate the maturation of nerve cells and axonal patterns in human fetal brain. Cell Tissue Res. 1998;291:433-43. 
88. Sanabria ER, Silva AV, Spreafico R, Cavalheiro EA. Damage, reorganization, and abnormal neocortical hyperexcitability in the pilocarpine model of temporal lobe epilepsy. Epilepsia. 2002;43(Suppl 5):96-106.

89. Shetty AK, Turner DA. Non-phosphorylated neurofilament protein immunoreactivity in adult and developing rat hippocampus: specificity and application in grafting studies. Brain Res. 1995;676:293-306.

90. Atik A, Cheong J, Harding R, Rees S, De Matteo R, Tolcos M. Impact of daily high-dose caffeine exposure on developing white matter of the immature ovine brain. Pediatr Res. 2014;76:54-63.

91. Maximova OA, Faucette LJ, Ward JM, Murphy BR, Pletnev AG. Cellular inflammatory response to flaviviruses in the central nervous system of a primate host. J Histochem Cytochem. 2009;57:973-89.

92. Goubran M, Hammond RR, de Ribaupierre S, Burneo JG, Mirsattari S, Steven DA, et al. Magnetic resonance imaging and histology correlation in the neocortex in temporal lobe epilepsy. Ann Neurol. 2015;77:237-50

93. Murray ME, Vemuri P, Preboske GM, Murphy MC, Schweitzer KJ, Parisi JE, et al. A quantitative postmortem MRI design sensitive to white matter hyper intensity differences and their relationship with underlying pathology. J Neuropathol Exp Neurol. 2012;71:1113-22.

94. Bachstetter AD, Rowe RK, Kaneko M, Goulding D, Lifshitz J, Van Eldik LJ. The p38a MAPK regulates microglial responsiveness to diffuse traumatic brain injury. J Neurosci. 2013;33:6143-53.

95. Bachstetter AD, Norris CM, Sompol P, Wilcock DM, Goulding D, Neltner $\mathrm{JH}$, et al. Early stage drug treatment that normalizes proinflammatory cytokine production attenuates synaptic dysfunction in a mouse model that exhibits age-dependent progression of Alzheimer's disease-related pathology. J Neurosci. 2012;32:10201-10.

96. Chen Z, Shin D, Chen S, Mikhail K, Hadass O, Tomlison BN, et al. Histological quantitation of brain injury using whole slide imaging: a pilot validation study in mice. PLoS ONE. 2014;9:e92133.

97. Dunn WD Jr, Gearing M, Park Y, Zhang L, Hanfelt J, Glass JD, et al. Applicability of digital analysis and imaging technology in neuropathology assessment. Neuropathology. 2016;36:270-82.

98. Wood TC, Simmons C, Hurley SA, Vernon AC, Torres J, Dell'Acqua F, et al. Whole-brain ex vivo quantitative MRI of the cuprizone mouse model. PeerJ. 2016:4:e2632.

99. Bachstetter AD, Van Eldik LJ, Schmitt FA, Neltner JH, Ighodaro ET, Webster SJ, et al. Disease-related microglia heterogeneity in the hippocampus of Alzheimer's disease, dementia with Lewy bodies, and hippocampal sclerosis of aging. Acta Neuropathol Commun. 2015;3:32.

100. Irwin DJ, Byrne MD, McMillan CT, Cooper F, Arnold SE, Lee EB, et al. Semi-automated digital image analysis of Pick's disease and TDP-43 proteinopathy. J Histochem Cytochem. 2016;64:54-66.

101. Josephs KA, Whitwell JL, Weigand SD, Murray ME, Tosakulwong N, Liesinger AM, et al. TDP-43 is a key player in the clinical features associated with Alzheimer's disease. Acta Neuropathol. 2014;127:811-24.

102. Mackenzie IR, Neumann M. Reappraisal of TDP-43 pathology in FTLD-U subtypes. Acta Neuropathol. 2017;134:79-96

103. Tan RH, Shepherd CE, Kril JJ, McCann H, McGeachie A, McGinley C, et al. Classification of FTLD-TDP cases into pathological subtypes using antibodies against phosphorylated and non-phosphorylated TDP43. Acta Neuropathol Commun. 2013;1:33.

104. Tan RH, Kril JJ, Fatima M, McGeachie A, McCann H, Shepherd C, et al. TDP-43 proteinopathies: pathological identification of brain regions differentiating clinical phenotypes. Brain. 2015;138:3110-22.

105. Huszar IN, Miller KL, Pallebage-Gamarallage M, Ansorge O, Mirfin C, Heinrich MP, et al. Pipeline for registering histological sections to MRI volumes [abstract]. In: 26th annual meeting of ISMRM; 2018 June 16-21; Paris, France.

106. Heinrich MP, Jenkinson M, Bhushan M, Matin T, Gleeson FV, Brady SM, et al. MIND: modality independent neighbourhood descriptor for multimodal deformable registration. Med Image Anal. 2012;16:1423-35.

107. Mollink J, Kleinnijenhuis M, Cappellen van Walsum AV, Sotiropoulos SN, Cottaar M, Mirfin C, et al. Evaluating fibre orientation dispersion in white matter: comparison of diffusion MRI, histology and polarized light imaging. Neuroimage. 2017;157:561-74.

108. Greve DN, Fischl B. Accurate and robust brain image alignment using boundary-based registration. Neuroimage. 2009;48:63-72.
109. O'Reilly JX, Beckmann CF, Tomassini V, Ramnani N, Johansen-Berg H. Distinct and overlapping functional zones in the cerebellum defined by resting state functional connectivity. Cereb Cortex. 2010;20:953-65.

110. Mori H, Yagishita A, Takeda T, Mizutani T. Symmetric temporal abnormalities on MR imaging in amyotrophic lateral sclerosis with dementia. Am J Neuroradiol. 2007;28:1511-6.

111. Wakana S, Jiang H, Nagae-Poetscher LM, van Zijl PC, Mori S. Fiber tract-based atlas of human white matter anatomy. Radiology. 2004;230:77-87.

112. Schulthess I, Gorges M, Müller H-P, Lulé D, Del Tredici K, Ludolph AC, et al. Functional connectivity changes resemble patterns of pTDP-43 pathology in amyotrophic lateral sclerosis. Sci Rep. 2016;6:38391.

113. Loewe K, Machts J, Kaufmann J, Petri S, Heinze H-J, Borgelt C, et al. Widespread temporo-occipital lobe dysfunction in amyotrophic lateral sclerosis. Sci Rep. 2017;7:40252.

114. Zhang Y, Fang T, Wang Y, Guo X, Alarefi A, Wang J, et al. Occipital cortical gyrification reductions associate with decreased functional connectivity in amyotrophic lateral sclerosis. Brain Imaging Behav. 2016;11:1-7.

115. Bede P, Bokde A, Elamin M, Byrne S, McLaughlin RL, Jordan N, et al. Grey matter correlates of clinical variables in amyotrophic lateral sclerosis (ALS): a neuroimaging study of ALS motor phenotype heterogeneity and cortical focality. J Neurol Neurosurg Psychiatry. 2013;84:766-73.

116. Mezzapesa DM, D’Errico E, Tortelli R, Distaso E, Cortese R, Tursi M, et al. Cortical thinning and clinical heterogeneity in amyotrophic lateral sclerosis. PLoS ONE. 2013;8:e80748.

117. Pagani M, Chio A, Valentini MC, Oberg J, Nobili F, Calvo A, et al. Functional pattern of brain FDG-PET in amyotrophic lateral sclerosis. Neurology. 2014;83:1067-74

118. Bourne RM, Bailey C, Johnston EW, Pye H, Heavey S, Whitaker $\mathrm{H}$, et al. Apparatus for histological validation of in vivo and ex vivo magnetic resonance imaging of the human prostate. Front Oncol. 2017;7:47.

119. Mac Donald CL, Dikranian K, Song SK, Bayly PV, Holtzman DM, Brody DL. Detection of traumatic axonal injury with diffusion tensor imaging in a mouse model of traumatic brain injury. Exp Neurol. 2007;205:116-31.

120. Aung WY, Mar S, Benzinger TLS. Diffusion tensor MRI as a biomarker in axonal and myelin damage. Imaging Med. 2013;5:427-40.

121. Dyakin W, Chen Y, Branch CA, Veeranna Yuan A, Rao M, et al. The contributions of myelin and axonal caliber to transverse relaxation time in shiverer and neurofilament-deficient mouse models. Neuroimage. 2010;51:1098-105.

122. Di Paola M, Phillips OR, Sanchez-Castaneda C, Di Pardo A, Maglione V, Caltagirone $\mathrm{C}$, et al. MRI measures of corpus callosum iron and myelin in early Huntington's disease. Hum Brain Mapp. 2014;35:3143-51.

123. Duyn JH, Schenck J. Contributions to magnetic susceptibility of brain tissue. NMR Biomed. 2017;30.

124. Wisnieff C, Ramanan S, Olesik J, Gauthier S, Wang Y, Pitt D. Quantitative susceptibility mapping (QSM) of white matter multiple sclerosis lesions: interpreting positive susceptibility and the presence of iron. Magn Reson Med. 2015;74:564-70.

\section{Submit your next manuscript to BioMed Central and we will help you at every step:}

- We accept pre-submission inquiries

- Our selector tool helps you to find the most relevant journal

- We provide round the clock customer support

- Convenient online submission

- Thorough peer review

- Inclusion in PubMed and all major indexing services

- Maximum visibility for your research

Submit your manuscript at www.biomedcentral.com/submit 